\title{
Role of mannitol metabolism in the pathogenicity of the necrotrophic fungus Alternaria brassicicola
}

\author{
Benoit Calmes ${ }^{1,2,3}$, Thomas Guillemette ${ }^{1,2,3 *}$, Lény Teyssier ${ }^{1,2,3}$, Benjamin Siegler $^{4}$, Sandrine Pigné1,2,3, \\ Anne Landreau ${ }^{5}$, Béatrice lacomi ${ }^{6}$, Rémi Lemoine ${ }^{7}$, Pascal Richomme ${ }^{5}$ and Philippe Simoneau ${ }^{1,2,3}$ \\ SFR 4207 QUASAV, UMR 1345 IRHS, Université d'Angers, Angers Cedex, France \\ 2 SFR 4207 QUASAV, INRA, UMR 1345 IRHS, Angers Cedex, France \\ ${ }^{3}$ SFR 4207 QUASAV, Agrocampus-Ouest, UMR 1345 IRHS, Angers Cedex, France \\ ${ }^{4}$ Plateforme d'Ingénierie et Analyses Moléculaires, Université d'Angers, Angers Cedex, France \\ ${ }^{5}$ SONAS EA 921, SFR 4207, QUASAV UFR des Sciences Pharmaceutiques et d'Ingénierie de la Santé, Université d'Angers, Angers Cedex, France \\ ${ }^{6}$ USAMV, Bucharest, Romania \\ Ecologie, Biologie des Interactions, UMR 7267 CNRS/Université de Poitiers, Poitiers, France
}

Edited by:

Adi Avni, Tel Aviv University, Israel

Reviewed by:

Mary B. Mudgett, Stanford

University, USA

Jane Glazebrook, University of

Minnesota-Twin Cities, USA

${ }^{*}$ Correspondence:

Thomas Guillemette, Faculté des Sciences, SFR 4207 QUASAV,

UMR 1345 IRHS, Université

d'Angers, 2 bd Lavoisier,

49045 Angers Cedex, France.

e-mail: thomas.guillemette@

univ-angers.fr
In this study, the physiological functions of fungal mannitol metabolism in the pathogenicity and protection against environmental stresses were investigated in the necrotrophic fungus Alternaria brassicicola. Mannitol metabolism was examined during infection of Brassica oleracea leaves by sequential HPLC quantification of the major soluble carbohydrates and expression analysis of genes encoding two proteins of mannitol metabolism, i.e., a mannitol dehydrogenase (AbMdh), and a mannitol-1-phosphate dehydrogenase ( $A b M p d)$. Knockout mutants deficient for $A b M d h$ or $A b M p d$ and a double mutant lacking both enzyme activities were constructed. Their capacity to cope with various oxidative and drought stresses and their pathogenic behavior were evaluated. Metabolic and gene expression profiling indicated an increase in mannitol production during plant infection. Depending on the mutants, distinct pathogenic processes, such as leaf and silique colonization, sporulation, survival on seeds, were impaired by comparison to the wild-type. This pathogenic alteration could be partly explained by the differential susceptibilities of mutants to oxidative and drought stresses. These results highlight the importance of mannitol metabolism with respect to the ability of $A$. brassicicola to efficiently accomplish key steps of its pathogenic life cycle.

Keywords: mannitol, Alternaria brassicicola, pathogenicity, null mutants, oxidative and drought stress, isothiocyanates, brassicicolin A

\section{INTRODUCTION}

Mannitol is a six-carbon non-cyclic sugar alcohol which appears to be widespread in the biosphere, with the noticeable exception of the animal kingdom. This polyol (i.e., alcohol containing multiple hydroxyl groups) is ubiquitous throughout the fungal kingdom and is considered as being the most abundant of all soluble carbohydrates within mycelia and fruit bodies (Lewis and Smith, 1967; Horer et al., 2001; Dulermo et al., 2009). In fungi, mannitol and its metabolism have been postulated to have a multitude of functions as either a carbohydrate reserve, in NADPH regeneration, in morphogenesis and conidiation or as a protection from environmental stress (Solomon et al., 2007). Furthermore, some studies have reported that mannitol has a role in pathogenicity of plant and animal pathogens (Chaturvedi et al., 1996a; Velez et al., 2008). Levels of mannitol were found to rise dramatically during plant infection by biotrophic or necrotrophic fungi and this accumulation was accompanied by increased expression of genes involved in the mannitol pathway (Voegele et al., 2005; Jobic et al., 2007; Dulermo et al., 2009).

Two hypotheses mainly emerged to explain the pathogenic significance of mannitol production by fungi. Firstly, fungal mannitol may be involved in the sequestration of carbohydrates from host. Since many plants are unable to metabolize mannitol, the conversion of plant hexoses into mannitol seems an ideal strategy for the fungal pathogen or mutualist, providing a means for fungi to store carbohydrates and reducing power in a form not accessible to the host (Ceccaroli et al., 2003; Dulermo et al., 2009). As in planta mannitol accumulation mainly occurred when conidiophores emerged, the latter authors suggest that this polyol could be necessary for spore survival or germination. Similar conclusions were suggested in the case of the biotrophic interaction between the rust fungus Uromyces fabae and its host plant Vicia faba, or during pathogenesis of Sclerotinia sclerotiorum, a B. cinerea-related necrotroph (Voegele et al., 2005; Jobic et al., 2007).

Secondly, mannitol is supposed to act as an antioxidant agent and protect fungal cells by quenching reactive oxygen species (ROS) produced by hosts in response to attack. Polyols can thus be powerful radical scavengers in vitro (Smirnoff and Cumbes, 1989; Voegele et al., 2005) and in vivo (Shen et al., 1997a,b). In the animal pathogen Cryptococcus neoformans, a mannitol low-producing mutant was hyper-susceptible to oxidative killing by normal human neutrophils and by cell-free oxidants, and was hypovirulent in mice (Chaturvedi et al., 1996a,b). 
Moreover, transgenic tobacco lines constitutively expressing a celery mannitol dehydrogenase $(\mathrm{MDH})$ or a plasma membrane mannitol transporter were shown to have enhanced resistance to pathogenic Alternaria species (Jennings et al., 2002; JuchauxCachau et al., 2007). These results suggested that both plantexpressed proteins supported the metabolism of fungal secreted mannitol, thus rendering the pathogen more susceptible to reactive oxygen-mediated plant defense. This hypothesis was further strengthened by the fact that the constitutive expression of the $\mathrm{MDH}$ transgene did not affect the pathogenicity of the nonmannitol-secreting fungal pathogen Cercospora nicotianae.

Mannitol metabolism in fungi was initially thought to be a cyclical process (Hult and Gatenbeck, 1978). In this cycle (Figure 1), mannitol-1-phosphate 5-dehydrogenase (MPD; EC 1.1.1.17) was proposed to reduce fructose 6-phosphate into mannitol-1-phosphate using the NADH cofactor, followed by dephosphorylation by a mannitol-1-phosphate phosphatase (MPP; EC 3.1.3.22), resulting in inorganic phosphate and mannitol. Mannitol would then be oxidized to fructose by mannitol dehydrogenase $\left(\mathrm{MDH}\right.$; EC 1.1.1.138) using the $\mathrm{NADP}^{+}$cofactor. Finally, fructose would be phosphorylated to fructose 6phosphate by a hexokinase (HX; EC 2.7.1.1). Dephosphorylation of mannitol-1-phosphate into mannitol via MPP was described as being irreversible. Consequently, the proposed cycle would go in one direction with MPD as the major biosynthetic enzyme and $\mathrm{MDH}$ as a catabolic enzyme. However, recent data based on gene disruption experiments indicated that mannitol metabolism is not a cyclical process (Solomon et al., 2006; Velez et al., 2007; Dulermo et al., 2010). According to these reports, mannitol synthesis and degradation were both severely impacted by the loss of MPD, while the deletion of MDH appeared to have a more limited effect. Moreover, the $m d h$ strains were found to be able to use mannitol as a sole carbon source, indicating that mannitol was not only catabolized by oxidation to fructose. Dulermo et al. (2010) recently reported the existence of a mannitol phosphorylation pathway in $B$. cinerea, suggesting that mannitol could be metabolized through mannitol-1-phosphate.

The behavior of $m p d$ and $m d h$ null strains in planta also questioned the importance of the mannitol pathway in fungal pathogenicity. Indeed, regardless of the fungus involved (A. alternata, $S$. nodorum or $B$. cinerea), the virulence of the $m p d$ and $m d h$ strains was not or partially compromised (Solomon et al., 2005, 2006; Velez et al., 2008; Dulermo et al., 2010). Nevertheless, mannitol was shown to be required for in planta sporulation, which is a crucial step in a polycyclic pathogen like $S$. nodorum (Solomon et al., 2005, 2006).

In this study, we investigated the role of the mannitol pathway in the plant necrotrophic fungus Alternaria brassicicola. This fungus causes black spot disease and is an economically important seed-borne fungal pathogen of Brassicaceae species. We isolated the genes encoding the MPD and MDH enzymes in A. brassicicola and used targeted gene disruption to create single and double mutants for each gene. We then explored the physiological functions of mannitol metabolism and, in particular, its involvement

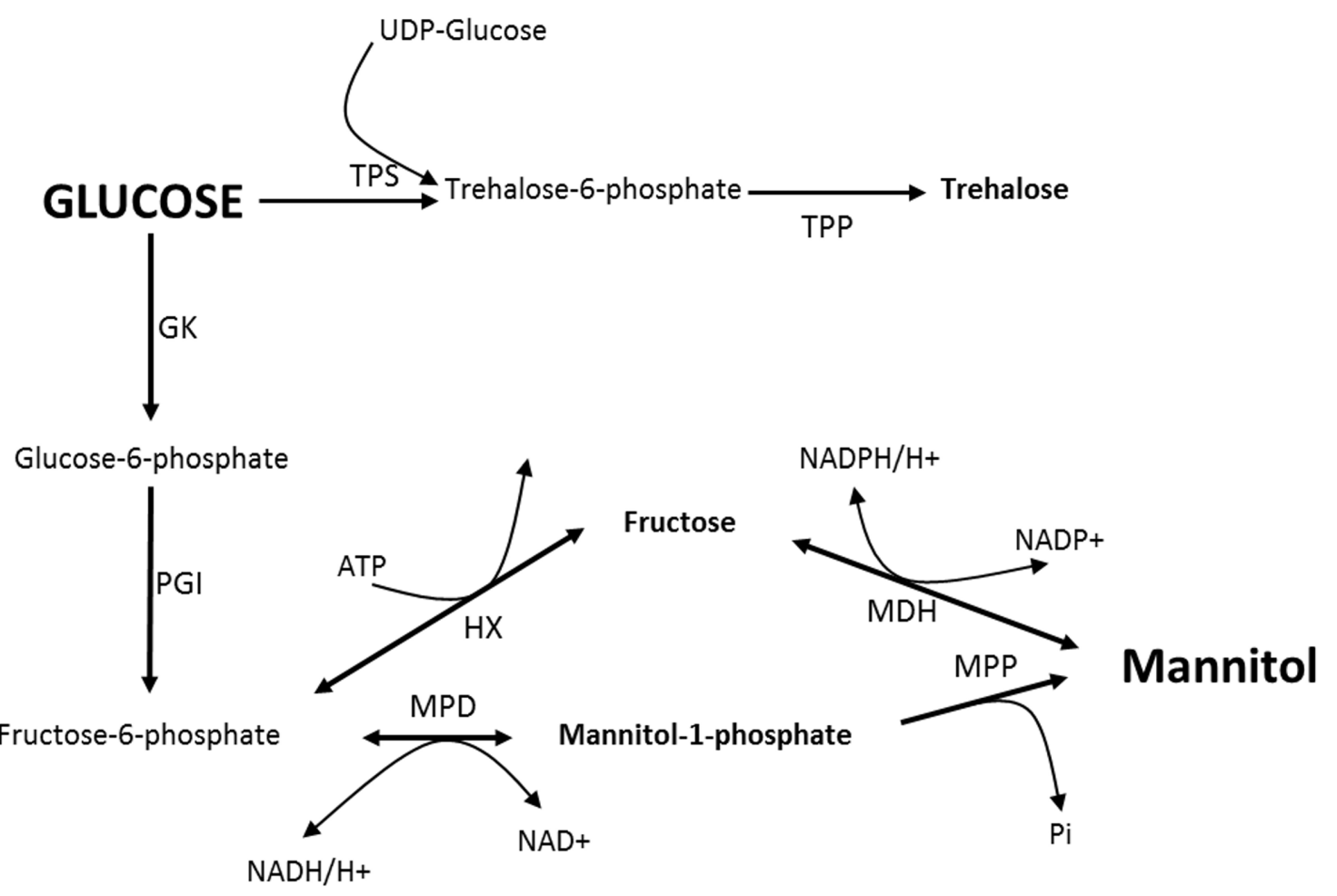

FIGURE 1 | Proposed mannitol cycle in fungi. MDH, Mannitol dehydrogenase; MPP, Mannitol-1-P phosphatase; MPD, Mannitol-1-P dehydrogenase; HX, Hexokinase; PGI, Phosphoglucose isomerase; GK, Glucokinase; TPS, Trehalose-6-P synthase; TPP, Trehalose-6-P phosphatase. 
in A. brassicicola pathogenicity and in the protection of fungal cells against defense compounds [like isothiocyanates (ITC)] and other environmental stresses.

\section{MATERIALS AND METHODS FUNGAL STRAINS AND GROWTH CONDITIONS}

The A. brassicicola wild-type strain Abra43 used in this study has previously been described (Dongo et al., 2009; Joubert et al., 2011a). For routine culture, A. brassicicola was grown and maintained on potato dextrose agar (PDA) or on Vogel minimal medium (Vogel, 1956). For osmotic stress experiments mycelia were grown on PEG-infused agar plates (Verslues et al., 2006). Colony diameters were measured daily and used for calculation of radial growth. The method based on microscale liquid cultivation (from conidial suspensions) and automated nephelometric recording of growth, followed by extraction of relevant variables (lag time and growth rate), was described by Joubert et al. (2010). To study the susceptibility of fungal strains to ITC, allyl-ITC (AlITC), benzyl-ITC (BzITC) or phenetyl-ITC (PhITC), were diluted from stock solutions prepared in acetone at the final desired concentrations $(2.5$ and $5 \mathrm{mM})$. ITC were purchased from Aldrich Chemical Co. (Milwaukee, WI). To study the effects of plant extracts on mannitol accumulation, plant extracts were prepared from primary leaves of tomato or radish as described by Ehrenshaft and Upchurch (1993) and sterilized by filtration through a $0.2-\mu \mathrm{m}$ nitrocellulose filter. Potato dextrose Broth (PDB) containing either 10\% (v/v) aqueous plantleaf extract or an equal volume of sterile distilled water were inoculated with conidia $\left(10^{5}\right.$ conidia/mL final concentration). Cultures were grown at $24^{\circ} \mathrm{C}$ with gentle agitation $(150 \mathrm{rpm})$ for 7 days.

\section{ANALYSIS OF CELL VIABILITY}

Propidium iodide (PI) was used as a cell viability marker. Viable cells with intact membranes exclude PI, whereas the membranes of dead and damaged cells are permeable to PI. Fungal suspensions were prepared on PDB with conidia for $10^{5}$ coni$\mathrm{dia} / \mathrm{mL}$ (final concentration). Non-germinated conidia and germinated conidia after $15 \mathrm{~h}$ of incubation $\left(150 \mathrm{rpm}, 24^{\circ} \mathrm{C}\right)$ were treated with $\mathrm{H}_{2} \mathrm{O}_{2}(8 \mathrm{mM})$ or Al-ITC $(5 \mathrm{mM})$. After $30 \mathrm{~min}$ of exposure, cells were washed twice with cold phosphate-buffered saline $\left(137 \mathrm{mM} \mathrm{NaCl}, 2.7 \mathrm{mM} \mathrm{KCl}, 4.3 \mathrm{mM} \mathrm{Na} 2 \mathrm{HPO}_{4} .7 \mathrm{H}_{2} \mathrm{O}\right.$, and $1.4 \mathrm{mM} \mathrm{KH}{ }_{2} \mathrm{PO}_{4}, \mathrm{pH} 7.4$ ) and then stained with PI $2 \mu \mathrm{g} / \mathrm{mL}$ (Sigma-Aldrich).

\section{GENERATION OF TARGETED GENE REPLACEMENT CONSTRUCTS AND FUNGAL TRANSFORMATION}

The gene replacement cassettes were generated using the doublejoint PCR procedure (Yu et al., 2004). The selectable marker inserted in the PCR constructs corresponded to the Hph gene cassette (1436 bp) from pCB1636 (Sweigard et al., 1995) or the Nat gene cassette (2150 bp) from pNR (Malonek et al., 2004) conferring resistance to hygromycin $\mathrm{B}$ and nourseotricin, respectively. The sets of primers used to amplify the $5^{\prime}$ and $3^{\prime}$ flanking regions of each targeted gene are presented in the Table 1. The doublejoint final PCR products were used to transform A. brassicicola protoplasts as described by Cho et al. (2006). The A. brassicicola wild-type Abra43 was used to obtain single hygromycin resistant transformant strains $\Delta a b m p d$ and $\Delta a b m d h$. The $\Delta a b m p d$ genotype was used to obtain $\Delta \Delta a b m p d$-abmdh hygromycin and nourseotricin resistant strains. Potential transformants were prescreened by PCR with relevant primer combinations (Table 1) to confirm integration of the replacement cassette at the targeted locus. Two putative gene replacement mutants for each construct were further purified by three rounds of single-spore isolation and then confirmed by Southern blot analysis.

\section{GENERATION OF FUSION PROTEIN CONSTRUCTS}

The Abmdh or Abmpd C-terminal GFP fusion constructs were generated by fusion PCR (Figure 2). Using A. brassicicola genomic DNA as a template, the respective ORFs and $3^{\prime}$ flanking regions were amplified with relevant primer combinations (Table 1). In parallel, a fragment containing the GFP cassettes and Hyg B cassettes were amplified from the plasmid pCT74 (Lorang et al., 2001). The resulting PCR fragments were mixed and subjected to second fusion PCR. A linker containing 3 glycine residues was introduced at the $3^{\prime}$ end of the respective ORFs to replace the stop codons. The final PCR products were transformed in the A. brassicicola wild-type to make AbMpd- and AbMdh-GFP fusion proteins. The transformants with expected genetic integration events were identified by PCR and Southern blot analyses (data not shown).

\section{NUCLEIC ACID ISOLATION AND ANALYSIS}

Genomic DNA extraction and Southern blot analysis were conducted as previously described by Joubert et al. (2011a). Total RNA extractions and amplification experiments were conducted as previously described (Joubert et al., 2011b) using specific primers for $A b M d h$ and $A b M p d$ genes (Table 1).

\section{INFECTION ASSAYS}

For plant infection assays on Brassica oleracea plants (var. Bartolo), $5 \mu \mathrm{L}$ drops of $A$. brassicicola conidia suspension $\left(10^{5}\right.$, $10^{4}$ or $10^{3}$ conidia/mL in water) were inoculated on leaves from 5 weeks-old plants. Inocula were symmetrically deposited on the left and right sides from the central vein. The plants were then maintained under saturating humidity (100\% relative humidity). Symptoms were observed and samples collected at 2, 4, 6 days post-inoculation (dpi) for the determination of major soluble carbohydrates contents and $A b M p d$ and $A b M d h$ expression analysis. For in planta sporulation assays, symptomatic tissues were sampled and vortexed for $30 \mathrm{~s}$ in water containing Tween $20(0.02 \%$, $\mathrm{v} / \mathrm{v})$. The concentration of the resulting conidia suspensions was estimated microscopically using a haemocytometer. For the microscopic analyses, B. oleracea leaf fragments were discolored, cleared and fungal structures were stained with solophenyl flavine 7GFE 500 (Ciba Specialty Chemicals, North Carolina, USA) as described by Hoch et al. (2005). Specimens were observed under a Leica fluorescent microscope (using $480 \mathrm{~nm}$ excitation and $527 \mathrm{~nm}$ emission).

\section{SEED CONTAMINATION ASSESSMENT}

Seed contamination assessments were estimated as described by Pochon et al. (2012). Two $2.5 \mu \mathrm{L}$ drops of an A. brassicicola conidial suspension $\left(1 \times 10^{5}\right.$ conidia $\mathrm{mL}^{-1}$ in water $)$ supplemented 
Table 1 | List of primers for the genes used in this study.

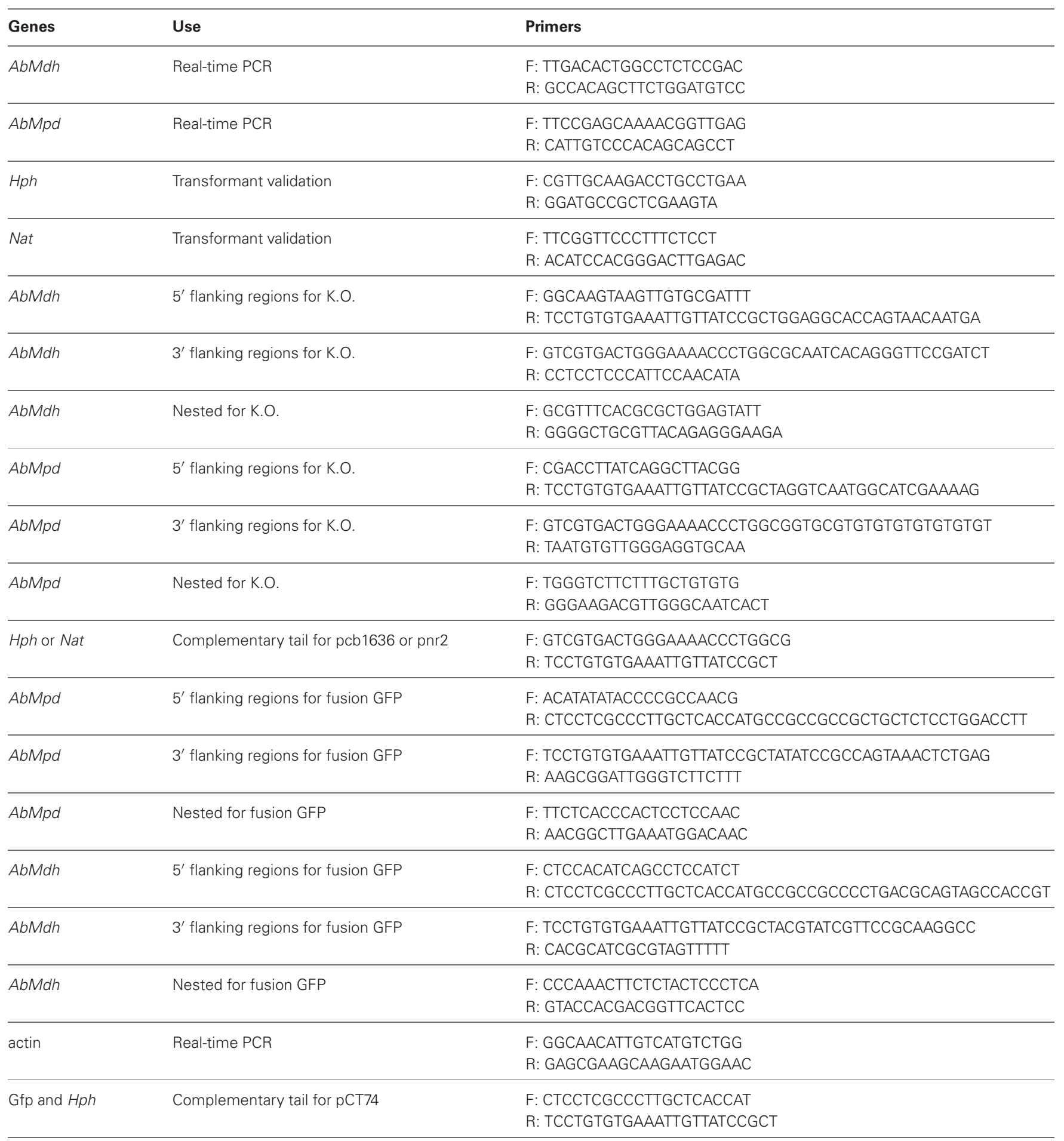

F, forward primer; $R$, reverse primer.

with $0.01 \%(\mathrm{v} / \mathrm{v})$ Tween 20 were placed on the five youngest siliques (one drop at the silique base and one in the middle) from 1-month-old A. thaliana (Ler) plants. At least five plants per fungal genotype were inoculated and the experiment was repeated twice. As a control for all experiments, two $2.5 \mu \mathrm{L}$ drops of a $0.01 \%(\mathrm{v} / \mathrm{v})$ Tween 20 solution were placed on five siliques of one plant. The plants were then maintained under saturating humidity for 2 days in the dark. Contaminated siliques were harvested $10 \mathrm{dpi}$. Inoculated or control siliques were dissected with sterile forceps and seeds were carefully harvested to avoid contact with the fungus potentially present on the outer surface of siliques. Seeds were incubated separately on PDA medium for 2 days. 


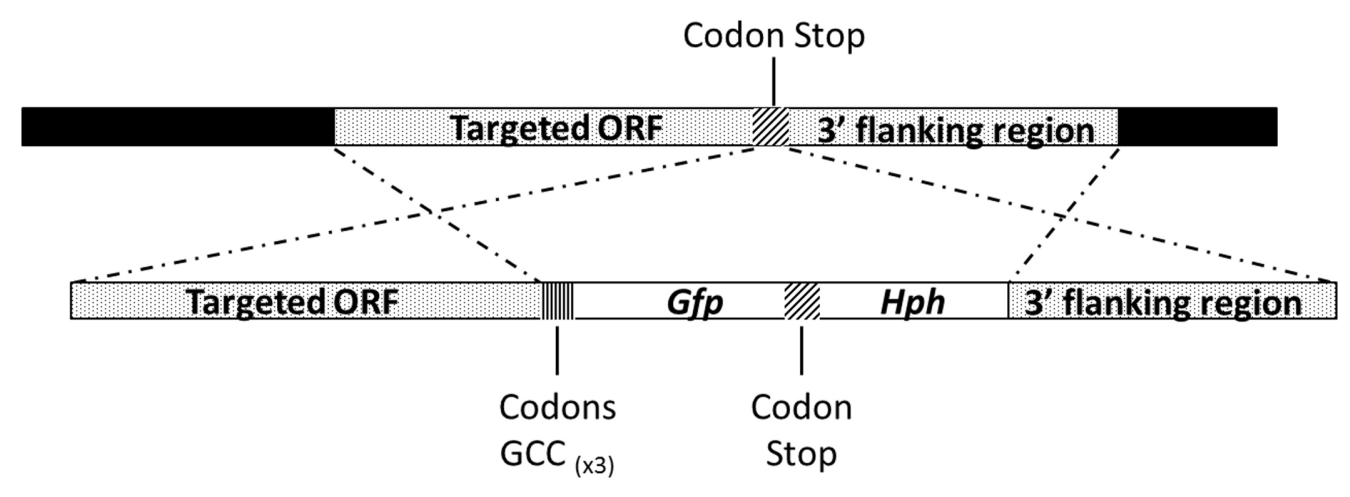

FIGURE 2 | Schematic representation of the AbMpd and AbMdh loci (dotted boxes) and replacement constructs with the GFP and HygB resistance (Hph) genes (white boxes).

A seed was considered as contaminated when incubation resulted in typical A. brassicicola colony development.

\section{CONSERVATION OF CONIDIA ON SEEDS}

$B$. oleracea seeds were artificially inoculated by incubation $(1 \mathrm{~h})$ in a conidia suspension $\left(5 \mathrm{~mL}\right.$ at $10^{5}$ conidia/mL). After removing the solution, the seeds were air-dried for $2 \mathrm{~h}$ and separated into two batches. The initial contamination rate was determined on one seed batch before storage. One seed per microplate well was placed in $300 \mu \mathrm{l}$ of PDB and fungal growth was recorded in a laser-based nephelometer. The mean lag time was calculated and representative of the initial seed infection rate. The second seed batch was stored in a dry dark place at $24^{\circ} \mathrm{C}$ for 6 months and processed as above. As the lag time was found to be directly proportional to the number of germinating conidia, the viability rate was estimated from the ratio between lag times before and after storage. This experiment was repeated twice for each fungal genotype.

\section{ENZYME ASSAYS}

Three-day-old cultures grown in PDB medium were harvested, submerged in liquid nitrogen for $5 \mathrm{~min}$ and stored at $-80^{\circ} \mathrm{C}$ until use. Extraction procedures for cell-free extracts were carried out at $4^{\circ} \mathrm{C}$ and MPD and MDH enzymatic activities were measured as described by Velez et al. (2007). For both enzymes, specific activities were defined as the $\mu$ mole of $\operatorname{NADP}(\mathrm{H})$ or $\operatorname{NAD}(\mathrm{H})$ oxidized per minute permg of protein. Three independent experiments were done for each sample.

\section{SUGAR AND SUGAR ALCOHOL EXTRACTION AND ANALYSIS}

Ethanolic extractions of cells from each sample used for hexose sugar and polyol measurements were performed as described by Stoop and Pharr (1993) with minor modifications. Dry powdered samples were suspended in $80 \%$ ethanol solution and incubated at $80^{\circ} \mathrm{C}$ for $5 \mathrm{~min}$. After $5 \mathrm{~min}$ of centrifugation at $1000 \mathrm{~g}$, the supernatants were recovered and pellets were re-extracted twice. Pooled ethanolic solutions were evaporated using a vacuum concentrator (speedVac UNIEQUIP), and residues were dissolved in sterile water or $\mathrm{D}_{2} \mathrm{O}$ for analysis. High performance liquid chromatography (HPLC) was performed on a Carbopac PA-1 column
(Dionex Corp., Sunnyvale, CA, USA) as described by Rosnoblet et al. (2007). For each sample, three independent experiments were done from separate cultures.

\section{DETECTION OF BRASSICICOLIN A FROM FUNGAL EXTRACTS}

Brassicicolin A was extracted as described by (Gloer et al., 1988) from cultures (minimal medium plus thiamine, Pedras et al., 1997) of Abra43 and $\Delta \Delta a b m p d-a b m d h$ strains. Each filtered culture broth $(1 \mathrm{~L})$ was extracted with ethyl acetate $(3 \times 300 \mathrm{ml})$, and the organic phase was dried over $\mathrm{MgSO}_{4}$ and evaporated to generate $25 \mathrm{mg}$ and $13.5 \mathrm{mg}$ of crude extracts from Abra43 and $\Delta \Delta a b m p d-a b m d h$ strains, respectively. Liquid chromatographymass spectrometry (LC/MS) was performed on each extract using a Bruker Esquire 3000 Plus electrospray ionization-ion trap mass spectrometer coupled with a Waters 2790 high performance liquid chromatography (HPLC-ESI-MS ${ }^{\mathrm{n}}$ ). Elution was carried out on an Hypersil RP18 column $(250 \times 4.6 \mathrm{~mm}, 5 \mu \mathrm{m}$, Termo $)$ using the following gradient: initial mobile phase $\mathrm{AcN} / \mathrm{H}_{2} \mathrm{O} \quad 0.01 \%$ formic acid 15/85 reaching 60/40 in $35 \mathrm{~min}$ and maintained for $10 \mathrm{~min}$ before reaching $100 / 0(\mathrm{v} / \mathrm{v})$ until $46 \mathrm{~min}$, with a flow rate of $1 \mathrm{~mL} / \mathrm{min}$. Only HPLC grade solvents were used. All samples were diluted in a solution of acetonitrile and filtered (UptiDisc ${ }^{\mathrm{TM}}$ PVDF $0.22 \mu \mathrm{m}$ ) prior to HPLC injection. They were analysed at $10 \mathrm{mg} / \mathrm{ml}$ concentration. The ESI parameters were as follows: solvent split ratio 1:9; nebulizer: 30 psi; dry gas $\left(\mathrm{N}_{2}\right)$ : 7 $\mathrm{L} / \mathrm{min}$; dry temperature: $340^{\circ} \mathrm{C}$; skim: $40 \mathrm{~V}$; trap drive: from 90 to 178 , octopole RF amplitude: from 144 to $210 \mathrm{Vpp}$; capillary exit: from -156 to -240 , capillary voltage $4500 \mathrm{~V}$. The ion trap mass spectrometer was run in negative ion scanning mode for $\mathrm{m} / \mathrm{z}$ ranging from 80 to $1500 . \mathrm{MS}^{\mathrm{n}}$ was performed at a fragmentation amplitude ranging from 0.8 to $2.0 \mathrm{~V}$ depending on the samples. Preparative thin-layer chromatography (PTLC) was carried out on silica gel 60F254 (0.25 mm, Merck) using MeoH/CHCL 3 (5/95) as eluent. This experiment was done twice from separate cultures of each fungal genotype.

\section{NMR ANALYSIS}

For nuclear magnetic resonance (NMR) metabolite analysis, $500 \mu \mathrm{l}$ of the $\mathrm{D}_{2} \mathrm{O}$-samples were transferred to a $5 \mathrm{~mm}$ NMR tube, then analyzed on a BRUKER Advance DRX $500 \mathrm{MHz}$ 
spectrometer equipped with a multinuclear QNP probe (Bruker, Wissembourg, France). Proton-decoupled ${ }^{13} \mathrm{C}$ NMR spectra (sweep width $=31450 \mathrm{~Hz}$ ) were recorded at $125 \mathrm{MHz}$ excitation frequency, 30-degrees pulse angle (6.5 $\mu$ s pulse duration) at $2 \mathrm{~s}$ intervals. The free induction decays were collected as $32 \mathrm{~K}$ data points and processed with a $1-2 \mathrm{~Hz}$ exponential line broadening for ${ }^{13} \mathrm{C}$ NMR. Maleic acid $\left(\delta_{\mathrm{CH}} 130.4 \mathrm{ppm}\right)$ was the external reference for chemical shifts. Identifications were made by comparison with spectra of pure known standards. For brassicicolin A, ${ }^{1} \mathrm{H}(500 \mathrm{MHz})$ and 2D NMR spectra (HMQC and COSY) were recorded in $\mathrm{CDCl}_{3}$ in a capillary probe (Bruker TXI $1.7 \mathrm{~mm}$ ) with chloroform resonances $\left(\delta_{\mathrm{H}} 7.28, \delta_{\mathrm{C}} 77.0 \mathrm{ppm}\right)$ as internal references. For each sample, NMR analysis was done twice.

\section{RESULTS}

CHARACTERIZATION OF Mpd AND Mdh GENES IN A. brassicicola AND GENERATION OF REPLACEMENT MUTANTS

The presumed Mpd and Mdh loci were identified by a homology search against the A. brassicicola genome assembly (http:// genome.jgi-psf.org/Altbr1/Altbr1.home.html) with genes previously described in A. alternata (Velez et al., 2007). AbMdh and AbMpd sequences (GenBank accession No JX403801 and
JX403800, respectively) consisted of 851 and 1173 nucleotides, respectively. Blast search on the whole genome sequence and Southern analyses suggested the presence of only one copy of each gene (Figure 3B). Among the putative regulatory elements identified on sequences upstream of the ATG, consensus sequences for the binding of transcription factors involved in response to thermal, osmotic and oxidative stresses (Msn2p/Msn4p, HsF2) were found on the two genes.

The resulting AbMdh protein belongs to the short-chain group of the dehydrogenase/reductase superfamily (Jornvall et al., 1995) and has 95,88 , and $75 \%$ identity to the corresponding proteins described in A. alternata, $S$. nodorum, and B. cinerea, respectively. The AbMpd amino acid sequence shares 92, 82, and 57\% identity with that of $A$. alternata, $S$. nodorum, and B. cinerea, respectively, and contains both NAD-interacting domains and a specific mannitol-1-phosphate dehydrogenase motif.

For each targeted gene, 2 replacement mutants (called $\Delta a b m d h 1-2, \Delta a b m p d 1-2)$ were generated by replacing the targeted ORF with a hygromycin B resistance cassette. Two $\Delta \Delta a b m p d-a b m d h 1-2$ double deletion mutants were then constructed by transforming the $\Delta a b m p d$ genotype with an $A b M d h$-replacement cassette containing a

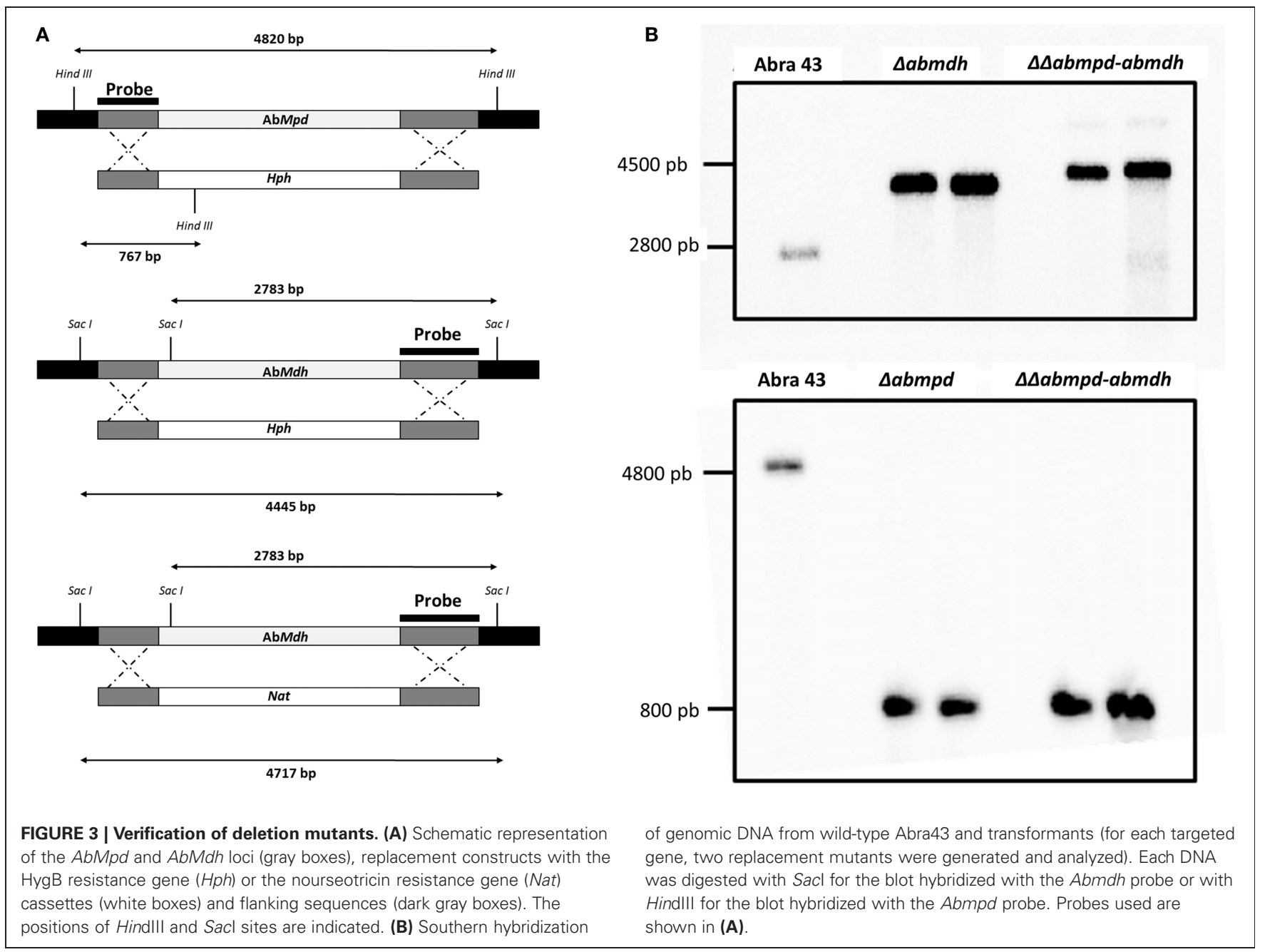


nourseothricin-resistance marker (Figure 3). In all further experiments, the phenotypic characters for transformants of the same genotype were not found to be significantly different.

\section{BIOCHEMICAL CHARACTERIZATION OF REPLACEMENT MUTANTS}

To confirm that gene inactivation impacted the enzyme activity, the transformants were grown in liquid culture and analysed for their ability to reduce fructose using NADPH as cofactor, or for fructose-6-phosphate conversion to mannitol-1-phosphate. Enzymatic assays (Table 2) confirmed that AbMdh and AbMpd deletions abolished mannitol dehydrogenase and mannitol-1phosphate dehydrogenase activities, respectively.

Table 2 | Enzyme activities related to mannitol metabolism in mycelia of Abra43 wild-type and mutant strains.

\begin{tabular}{|c|c|c|}
\hline Strains & $\begin{array}{l}\text { MDH activity } \\
\mu \mathrm{mol} \mathrm{min}^{-1} \mathrm{mg} \\
\text { of protein }\end{array}$ & $\begin{array}{l}\text { MPD activity } \\
\mu \mathrm{mol} \mathrm{min}^{-1} \mathrm{mg} \\
\text { of protein }\end{array}$ \\
\hline Abra43 & $3.7 \pm 0.16$ & $5.1 \pm 0.85$ \\
\hline$\Delta a b m p d$ & $3.7 \pm 0.03$ & 0 \\
\hline$\Delta a b m d h$ & 0 & $5.6 \pm 0.35$ \\
\hline$\Delta \Delta a b m p d-a b m d h$ & 0 & 0 \\
\hline
\end{tabular}

$\mathrm{MDH}$ activity was measured in extracts as the rate of mannitol-dependent conversion of $\mathrm{NAD}^{+}$to $\mathrm{NADH}$ and MPD activity was measured in extracts as the rate of mannitol-dependent conversion of NADP+ to NADPH (U). Data represent the means of three independent experiments.
The effects of the $\Delta a b m d h, \Delta a b m p d$, and $\Delta \Delta a b m p d-a b m d h$ mutations on the accumulation of sugars and sugar alcohols were estimated by ${ }^{13} \mathrm{C}$ NMR. Ethanolic extracts of sporulating mycelium grown in synthetic Vogel medium with glucose $(2 \%)$ were obtained and analysed (Figure 4A). The wild-type and $\Delta a b m p d$ extracts exhibited similar sugar profiles. However, lower amounts of mannitol and higher amounts of trehalose were found in the latter genotype. Conversely, the ${ }^{13} \mathrm{C}-\mathrm{NMR}$ spectra of $\Delta a b m d h$ mutants were dominated by mannitol resonances. Mannitol was absent in extracts from $\Delta \Delta a b m p d-a b m d h$ mutants in which trehalose and glycerol appeared to be the major compounds. Quantitative estimations of the mannitol content in the different genotypes during in vitro development were obtained by HPLC analysis of extracts from mature conidia and young non-sporulating mycelia (Figure 4B). While the wild-type accumulated nearly the same amount of mannitol in conidia and mycelia, this polyol was exclusively detected in conidia of the $\Delta a b m p d$ mutants. By contrast, the $\Delta a b m d h$ mutants preferentially accumulated mannitol in mycelia. No mannitol was detected in either conidia or young mycelia ( $30 \mathrm{~h}$ post germination) from the $\Delta \Delta a b m p d$-abmdh mutants. However, traces of mannitol were detected from the double deletion strains in 1-week-old cultures (data not shown).

\section{SUSCEPTIBILITY OF REPLACEMENT MUTANTS TO STRESS CONDITIONS}

Mannitol has been proposed to act as a potent protective metabolite against oxidative stress. As the mannitol contents of conidia

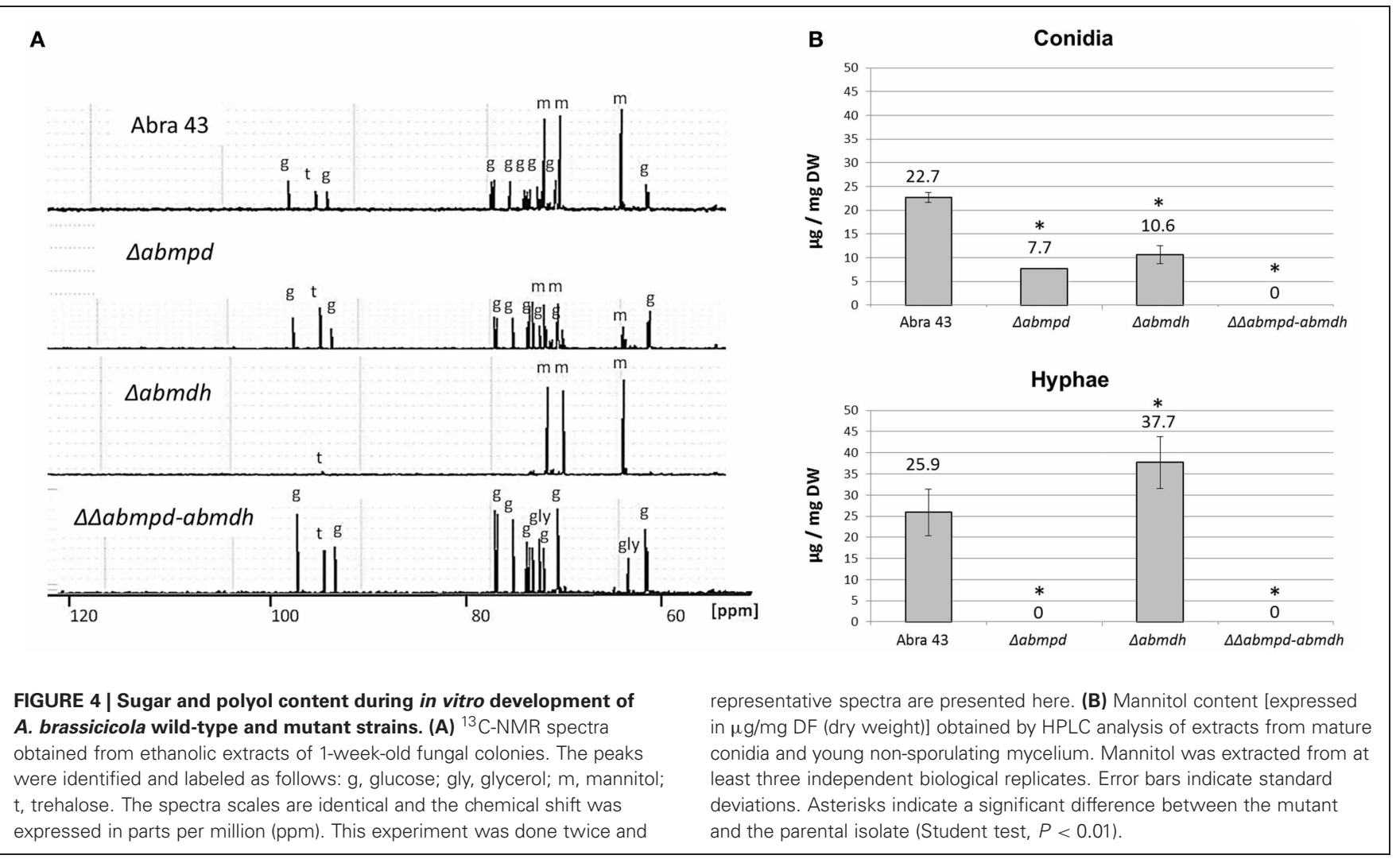


and mycelia from the different mutant genotypes were significantly different from that of the wild-type, the effects of $A b M p d$ and $A b M d h$ inactivation in $A$. brassicicola on conidia germination and initial mycelium growth in the presence of oxidative stressors were examined. Analysis of nephelometric growth curves revealed that under non-stress conditions (PDB medium), no significant phenotypic differences in conidia germination (based on the lag time parameter) or mycelium growth (based on the maximum growth rate parameter) were detected in any of the tested mutants as compared to the wild-type (data not shown). By contrast, $\Delta a b m d h$ and $\Delta a b m p d$ mutants, and to a lesser extent $\Delta \Delta a b m p d-a b m d h$ mutants, were far more susceptible than the wild-type to $1 \mathrm{mM} \mathrm{H} \mathrm{O}_{2}$ treatments (Figure 5A). The $\Delta a b m p d$ and $\Delta a b m p d-a b m d h$ mutants were also more susceptible to exposure to the superoxide-generating compound menadione than the wild-type and $\Delta a b m d h$ genotypes (Figure 5B). As some brassicaceous defense metabolites have antifungal properties that might be at least partially linked to their capacity to generate oxidative stress (Sellam et al., 2007a), the susceptibility to allyl-ITC (Al-ITC), benzyl-ITC (Bz-ITC), and phenetyl-ITC (Ph-ITC) and brassinin were investigated (Figures 5C-F). A genotype susceptibility pattern similar to that obtained with menadione was observed in the presence of $5 \mathrm{mM}$ Al-ITC, $5 \mathrm{mM} \mathrm{Bz}$-ITC, $10 \mathrm{mM}$ $\mathrm{Ph}$-ITC and $200 \mu \mathrm{m}$ brassinin. Note that after $24 \mathrm{~h}$ of exposure to ITC, the mannitol content of wild-type mycelia was 1.4-fold higher than in control and a 3 -fold increased expression

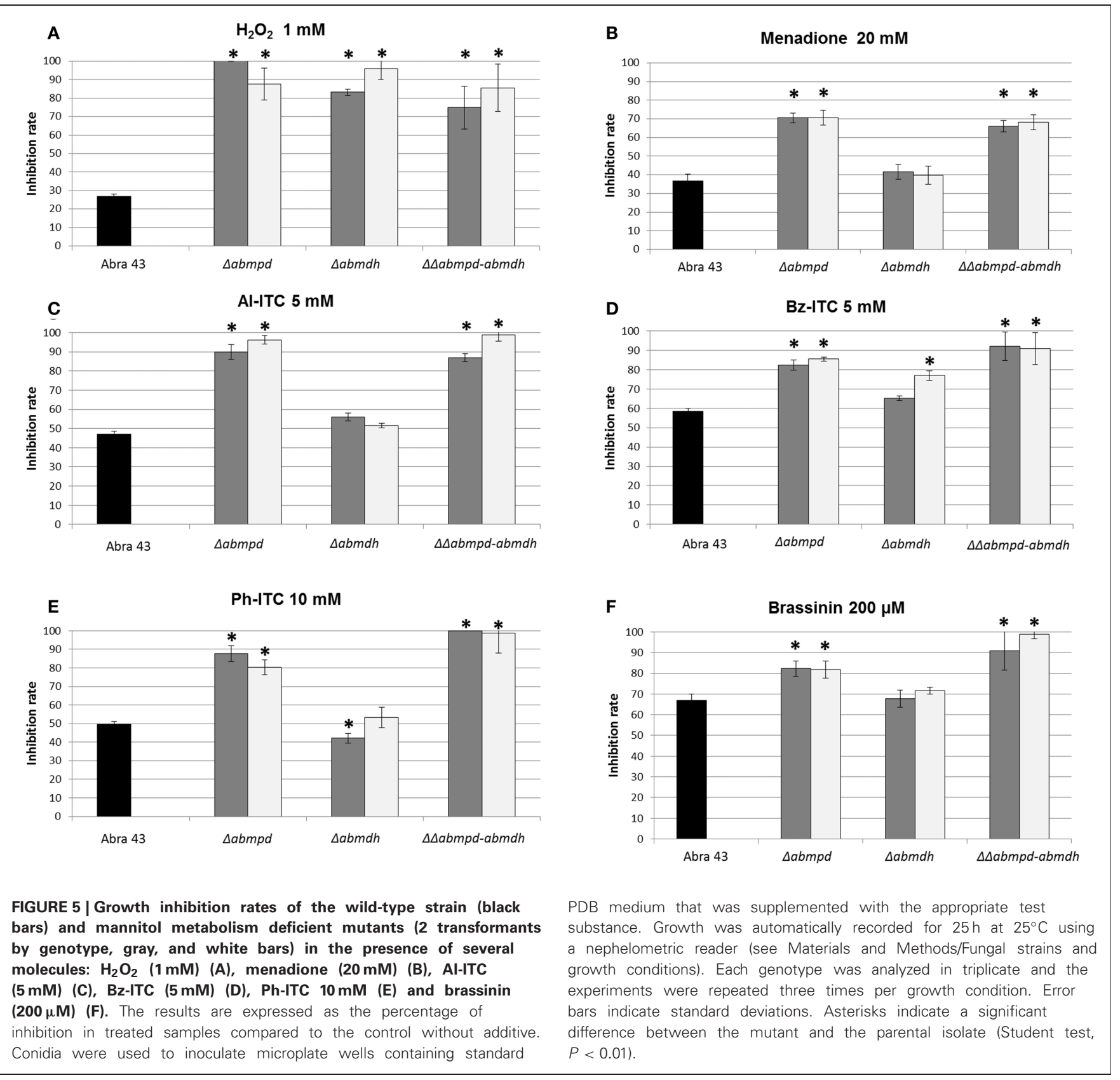


of $A b M p d$ was recorded in cultures after $3 \mathrm{~h}$ of ITC treatment, while the $A b M d h$ expression level remained unchanged (data not shown).

Based on these susceptibility patterns, comparing the response of the four genotypes to $\mathrm{H}_{2} \mathrm{O}_{2}$ on one hand and to menadione or ITC on the other suggested different modes of action for these oxidants. This was further supported by comparing the effects on the cell viability of non-germinated and germinated conidia of low-time exposure to $\mathrm{H}_{2} \mathrm{O}_{2}$ and Al-ITC at their respective $\mathrm{IC}_{50}$ (Figure 6). On germinated conidia, treatment with Al-ITC resulted in PI-positive hyphae suggesting a loss of cell viability. No PI staining was observed in non-germinated conidia exposed to Al-ITC. Conversely, $\mathrm{H}_{2} \mathrm{O}_{2}$ caused cell death in non-germinated or germinated conidia.

Besides its potential role as antioxidant molecule, mannitol has been proposed to provide protection against drought stress (Dijksterhuis and de Vries, 2006). Media with different water potentials (from $-0.25 \mathrm{MPa}$ to $-1.7 \mathrm{MPa}$ ) were prepared using a PEG-infused plate protocol (Verslues et al., 2006), inoculated with the different $A$. brassicicola genotypes and radial growth was recorded after 15 days of incubation. A shown in Figure 7, strains lacking functional MPD ( $\triangle a b m p d$ and $\Delta \Delta a b m p d$-abmdh) were much more susceptible to low water potential treatments than the wild-type. Conversely, $\Delta a b m d h$ mutants were more tolerant than the wild-type.

\section{MANNITOL METABOLISM DURING PLANT COLONIZATION}

The HPLC profiles of the major soluble carbohydrates present over the course of $A$. brassicicola infection on $B$. oleracea leaves were established (Figure 8A). At $2 \mathrm{dpi}$, the wild-type strain produced characteristic appressoria-like structures at the tips of germ tubes in contact with the leaf epidermis (Figure 8B). Small necrotic symptoms were observed at $4 \mathrm{dpi}$, and they continued to expand into large typical necrotic areas surrounded by chlorotic halos at $6 \mathrm{dpi}$. At this infection stage, necrotic spots exhibited dense conidia formation on the surface. The HPLC analysis showed that, while the mannitol level remained below the detection limit in control plants, mannitol accumulated throughout infection and revealed a twenty-fold increase from 2 dpi to 4 dpi. A small decrease in mannitol was then observed at $6 \mathrm{dpi}$ when sporulation was abundant. However, the mannitol level at this stage was still significantly higher than that detected at 2 dpi. Trehalose was only detected at low levels at 4 dpi and 6 dpi. Sucrose, which was the major carbohydrate in control noninoculated plant samples, quickly decreased to undetectable levels at 4 dpi.

The expression of $A b M d h$ and $A b M p d$ during infection was also examined (Figure 8C). At $2 \mathrm{dpi}$, the expression level of AbMpd increased and remained higher than during in vitro growth over the time course of the experiment. Increased $A b M d h$ expression was observed at a later stage and reached 3.5-fold its basal expression level at $6 \mathrm{dpi}$. These results are consistent with the metabolic profiling, which indicated a prevalence of mannitol production during plant infection. In order to follow the in vitro and in planta spatial expression patterns of both genes, strains expressing AbMpd and AbMdh, under the control of their own promoter and fused at their carboxy-terminal end to SGFP, were engineered. None of the transformants showed visible phenotypic changes discernible from the wild-type except for expression of green fluorescence (data not shown). In both strains, the fluorescence signal was detected in mature conidia and in the young germ tubes (Figure 9). In the in vitro-produced mature hyphae, the signal was still detectable but was much weaker in the AbMpd-GFP strain than in the AbMdh-GFP strain.

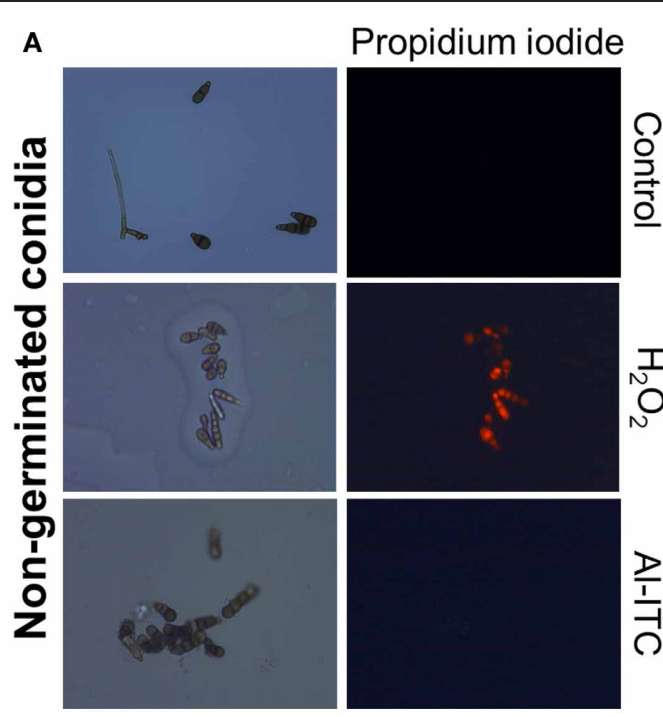

FIGURE 6 | Differential effect of $\mathrm{H}_{2} \mathrm{O}_{2}$ or AI-ITC on the viability of the $A$. brassicicola wild-type strain at different physiological stages.

Non-germinated conidia (A) and germinated conidia after $15 \mathrm{~h}$ of incubation

(B) were treated with $\mathrm{H}_{2} \mathrm{O}_{2}(8 \mathrm{mM})$ or Al-ITC $(5 \mathrm{mM})$ for 30 min or untreated
B

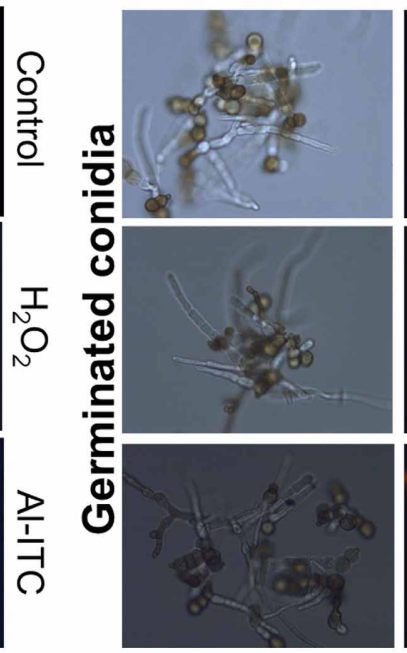

Propidium iodide

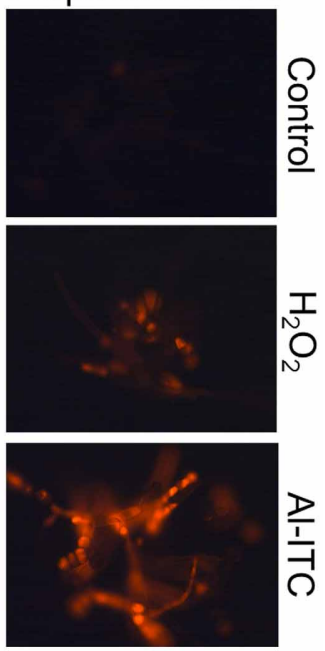

(control) before observation. Suspensions were labeled with propidium iodide, which reveals dead cells, before fluorescence microscopy examination. This experiment was done twice with at least 100 spores or germlings and representative pictures are presented here. 


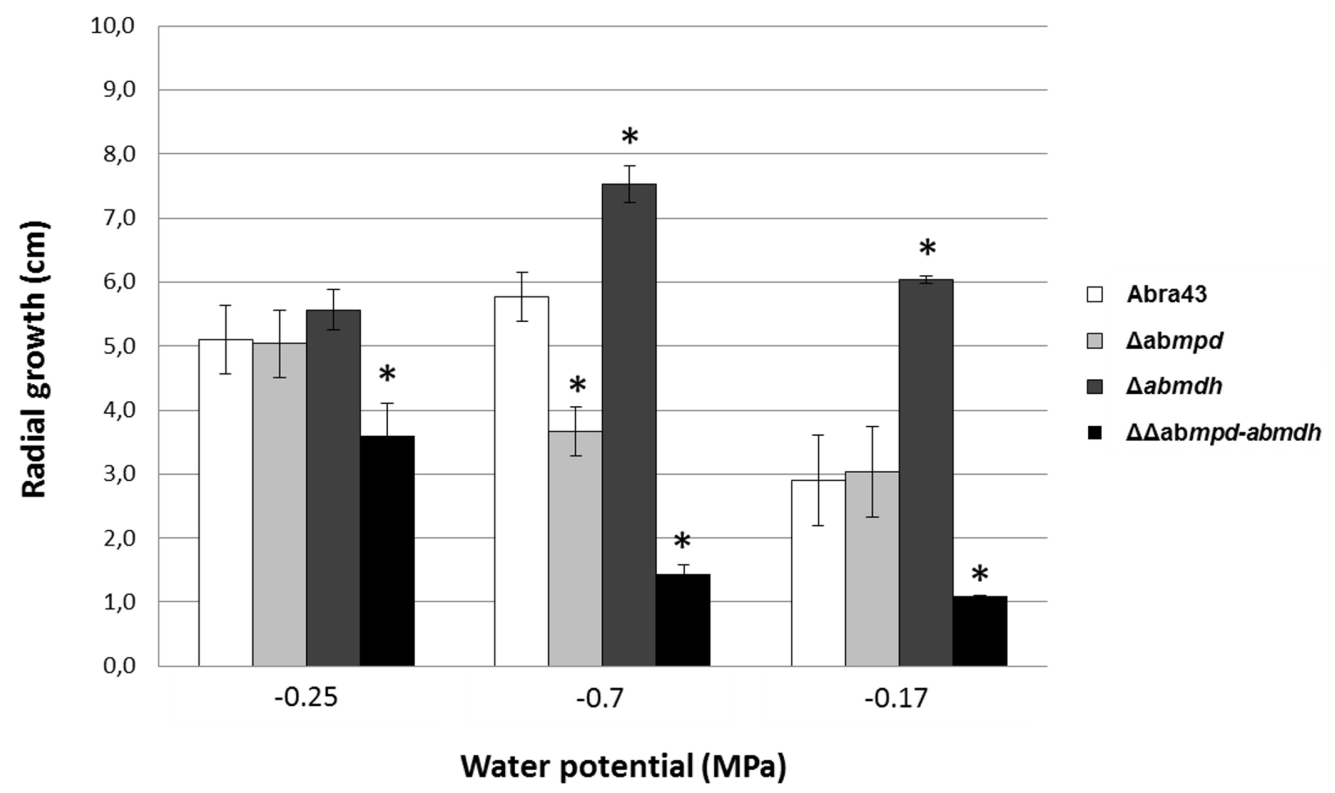

FIGURE 7 | Effect of low water potential treatments on the mycelium radial growth of the $A$. brassicicola wild-type strain and mannitol-metabolism mutants. In vitro growth tests were carried out on PEG-infused plates (Verslues et al., 2006). Colony diameters (cm) were measured after 15 days of incubation at $24^{\circ} \mathrm{C}$. Values are the means of three replicates. Error bars indicate standard deviations. Asterisks indicate a significant difference between the mutant and the parental isolate (Student test, $P<0.01$ ).
Consistent with the in planta expression patterns, GFP fluorescence appeared to be stronger in the mutant during host plant infection. In AbMpd- and AbMdh-GFP fusion mutants, fluorescence intensity increased dramatically during the in vitro or in planta conidiation process, reaching a maximum in young conidia (Figure 9).

To test the hypothesis that host plants would elicit changes in fungal mannitol production, the $A$. brassicicola wild-type strain was cultured for 7 days in the presence and absence of leaf extracts from host (B. oleracea) or non-host (Solanum lycopersicum) plants. The amount of mannitol in the fungal mycelia was then determined by HPLC (Figure 10). Fungal growth was essentially unaffected by plant extracts (data not shown). A. brassicicola responded to the presence of host plant extracts by accumulating significantly higher levels of mannitol as compared to the amount detected in control culture or in the presence of non-host extract.

\section{PATHOGENIC BEHAVIOR OF REPLACEMENT MUTANTS ON VEGETATIVE ORGANS}

The accumulation of mannitol throughout infection of B. oleracea by $A$. brassicicola and the increased susceptibility of mannitol biosynthesis mutants to oxidative stress prompted us to comparatively evaluate the pathogenicity of the different fungal genotypes. The wild-type and $\Delta a b m d h, \Delta a b m p d$, and $\Delta \Delta a b m p d-a b m d h$ mutants were all able to produce typical symptoms (Figure 11A). However, as judged from the lesion sizes at low inoculum charge, significant decreases in aggressiveness (up to $85 \%$ that of the wild-type at $10^{3}$ conidia per $\mathrm{ml}$ ) were recorded for the $\Delta \Delta a b m p d-a b m d h$ mutants and to lesser extent for the $\Delta a b m d h$ and $\Delta a b m p d$ mutants. Closer inspection of symptoms suggested that weak in planta sporulation occurred on necrosis obtained after inoculation with $A b M d h$ deficient mutants (Figure 11B). This was confirmed by measuring the quantity of conidia produced per $\mathrm{mm}^{2}$ of necrotic tissue. All genotypes produced significantly fewer conidia in planta than the wild-type, with a $90 \%$ reduction for $\Delta a b m d h$ mutants.

To check whether the lower aggressiveness of mutants could be correlated with in planta spore germination and/or plant tissue penetration defects, observation of solophenyl stained samples at 1 dpi was performed to quantify germinated conidia and appressoria-like structures at the plant surface. Although nearly $95 \%$ of conidia from all genotypes were germinated at this time, only 5 and $20 \%$ had differentiated infection structures in samples inoculated with the $\Delta \Delta a b m p d-a b m d h$ and the $\Delta a b m p d$ mutants, respectively, vs. $40 \%$ for samples inoculated with the wild-type or $\Delta a b m d h$ mutants. Following host penetration, the fungus has to produce necrotic factors to progress within infected tissues. Brassicicolin A, a fungal metabolite considered as being a mannitol derivative, represents a potent necrotic toxin produced by A. brassicicola (Pedras et al., 2009). We hypothesized that the weak virulence in mutants lacking mannitol may also be explained by the absence of brassicicolin A synthesis. To test this hypothesis, ethyl acetate extracts of culture filtrates from submerged cultures of $A$. brassicicola wild-type and $\Delta \Delta a b m p d$-abmdh strains were analysed by HPLC-UV-MS. The resulting total ion chromatograms (TIC) of both extracts revealed a major metabolite at $R_{\mathrm{t}}=39$ min (Figures 12A,B) exhibiting the quasimolecular ion $[\mathrm{M}-\mathrm{H}]^{-}(m / z 683)$ of brassicicolin $\mathrm{A}\left(\mathrm{C}_{32} \mathrm{H}_{48} \mathrm{~N}_{2} \mathrm{O}_{14}\right)$, whereas typical MS/MS fragments $(1 \mathrm{~V})$ were recorded at $\mathrm{m} / z, 565$ (loss 


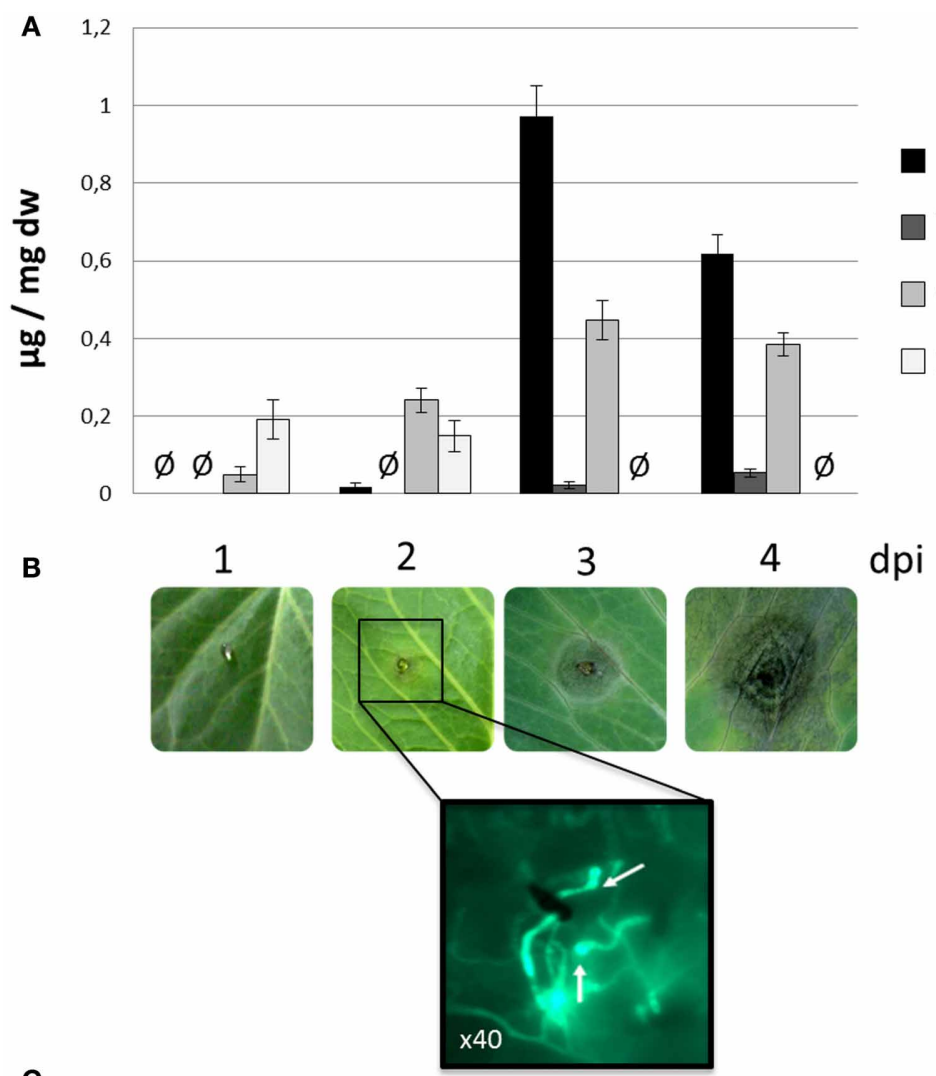

C

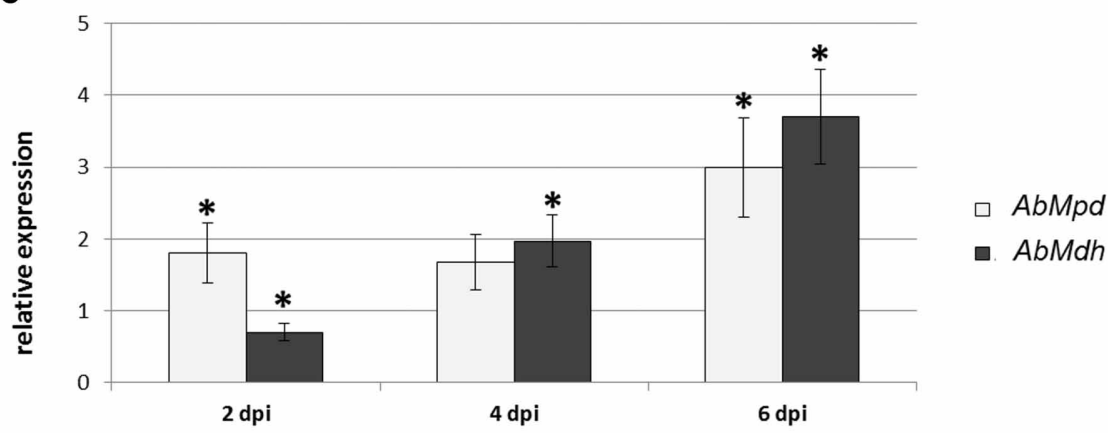

FIGURE 8 | Mannitol metabolism during plant colonization. (A) Major soluble carbohydrate concentrations (expressed in $\mu \mathrm{g} / \mathrm{mg}$ DF) measured by HPLC during the infection kinetics of the $A$. brassicicola wild-type strain on Brassica oleracea. Undetected sugars are represented by the symbol $\varnothing$. Three independent experiments were done. (B) Progression of symptoms on a B. oleracea leaf inoculated with the Abra43 strain and microscopic observations of the infection structures at 2 dpi. Inoculated plant tissue fragments were collected at 2 dpi and stained with solophenyl flavine for fluorescence microscopy observations. Appressoria-like structures are indicated by white arrows. (C) Quantitative
RT-PCR results for the expression of AbMpd (white bars) and AbMdh (dark gray bars) during the infection kinetics of $A$. brassicicola wild-type strain on $B$. oleracea. For each gene, expression induction is represented as a ratio of its relative expression at 2, 4, and $6 \mathrm{dpi}$ (studied gene transcript abundance/actin transcript abundance) in each inoculated sample to its relative expression in free-living fungal control cultures. The experiment was performed twice on biologically independent samples with three technical replicates. Error bars indicate standard deviations and asterisks indicate a relative expression significantly different from 1 (Student test, $P<0.01$ ). of two acetyl units) and 473 (loss of one $\alpha$-hydroxyisovaleryl unit together with one $\alpha$-isocyanoisovaleryl unit) (data not shown). The corresponding compound, which seems to accumulate in the same amounts in both strains, was purified through preparative TLC from the wild-type extract and analyzed $\left({ }^{1} \mathrm{H}\right.$ NMR, COSY and HMQC) in a capillary NMR probe (500 MHz). The resulting data (data not shown) were also in full agreement with former measurements obtained by Gloer et al. (1988) for this mixture of stereoisomers and confirmed that the isolated compound was brassicicolin A. We concluded that brassicicolin A was present in organic extracts from the culture broths of both the wild-type strain and the $\Delta \Delta a b m p d-a b m d h$ mutant and that the attenuated virulence of mannitol-deficient mutants could not be linked to the loss of brassicicolin A production. 

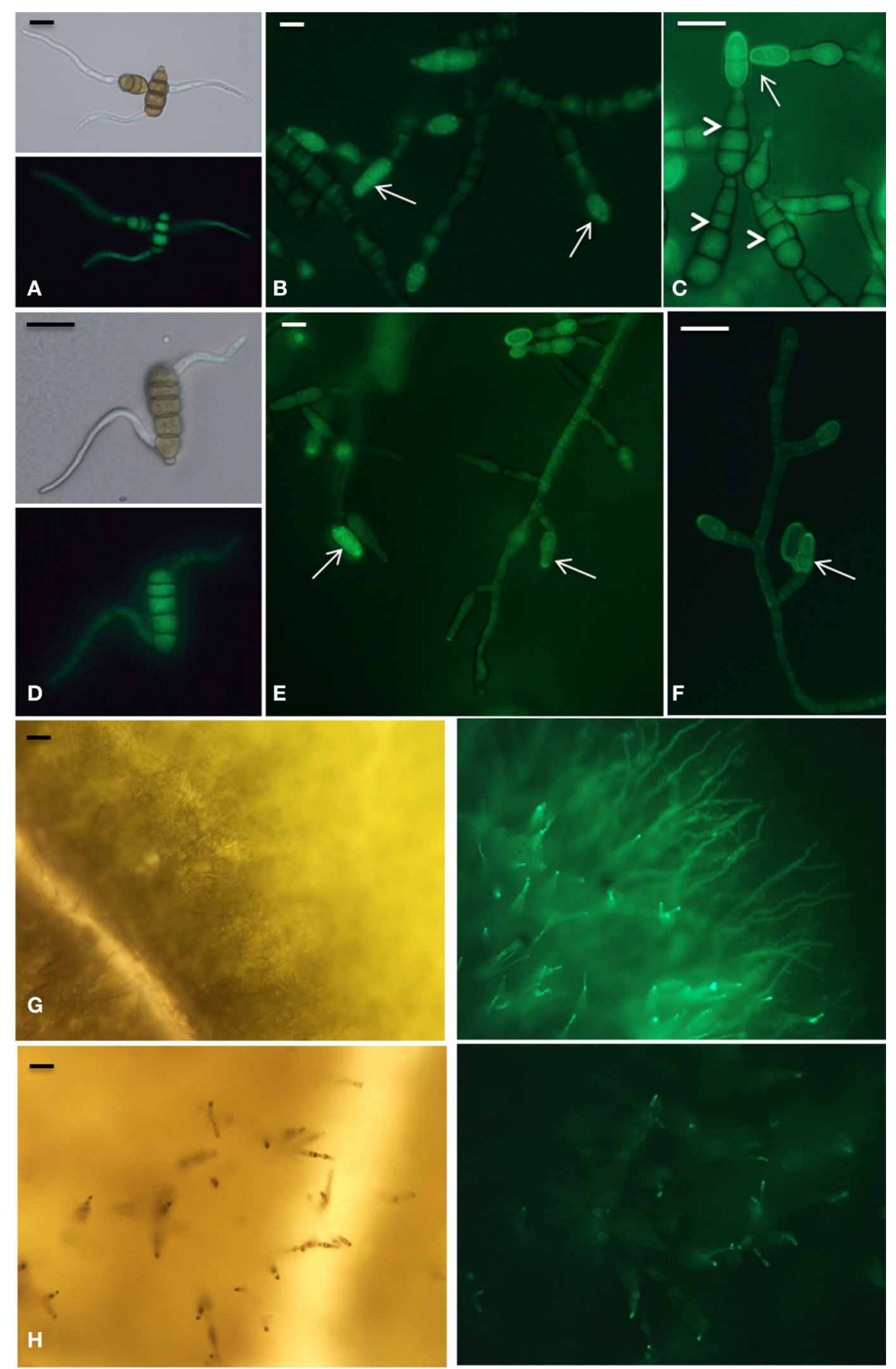

FIGURE 9 | Developmental phase-specific expression of AbMpd and AbMdh. All panels are microscopy images of GFP expression in

A. brassicicola strains expressing AbMdh (panels $\mathbf{A}, \mathbf{B}, \mathbf{C}$, and $\mathbf{G}$ ) and AbMpd (panels $\mathbf{D}, \mathbf{E}, \mathbf{F}$, and $\mathbf{H}$ ), under the control of their own promoter and fused at their carboxy-terminal end to SGFP. (A) and (D) Early germination stage $5 \mathrm{~h}$ after transfer to a solid PDA medium. Scale bars $=$ $10 \mu \mathrm{m}$. (B, C,E, and $\mathbf{F})$ Mycelia grown for $72 \mathrm{~h}$ on a PDA medium. At this stage, hyphae started to differentiate into conidiophores, leading to the production of young (arrow) and mature (arrowhead) conidia. Scale bars = $10 \mu \mathrm{m}$. (G) and (H) Fungal growth 6 days after inoculation of B. oleracea leaves. At this time, the fluorescence signal increased in aerial hyphae during the conidiation process. The right part corresponds to fluorescence microscopy and the left part to bright-field microscopy. Scale bars = $30 \mu \mathrm{m}$.

\section{PATHOGENIC BEHAVIOR OF REPLACEMENT MUTANTS ON REPRODUCTIVE ORGANS}

A. brassicicola is a seed-borne pathogen and has potential for long-term survival on dry seeds. As the differential abilities of the mutants to overcome a water potential stress have been observed, their capacity to survive on artificially contaminated seeds during storage was examined. After 6 months of storage, the percentage of viability was estimated using laser nephelometry growth 


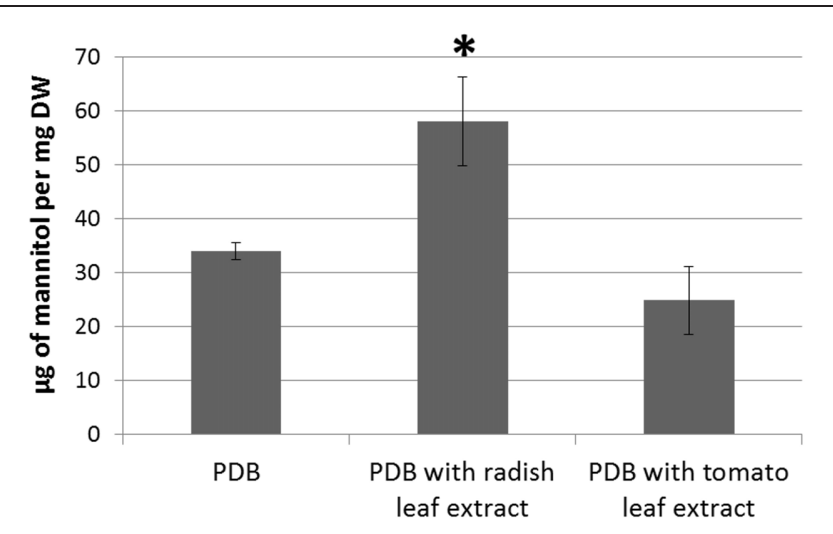

FIGURE 10 | HPLC assay of intracellular mannitol extracted from the mycelium of the wild-type genotype Abra43 grown in PDB supplemented or not with tomato or radish leaf extract $(10 \% \mathrm{v} / \mathrm{v})$ for 7 days. Data represent the means of three independent experiments and error bars indicate standard deviations. Asterisks indicate a significant difference from the control culture conditions (PDB only) using the Student test $(P<0.01)$

curves on the basis of $\Delta$ lag time values. As shown in Figure 13A, $\Delta \Delta a b m p d-a b m d h$ mutant survival was significantly lower than that of other tested genotypes. Similarly, the ability of A. brassicicola to efficiently infect seeds has been correlated with their capacity to cope with severe stress conditions consecutive to gradual dehydration of maturating reproductive organs (IacomiVasilescu et al., 2008). Using the model pathosystem recently described for investigating seed transmission in Arabidopsis plants (Pochon et al., 2012), the abilities of the mutants to transmit to seeds were compared with that of the wild-type. As shown in Figure 13B, the transmission capacity to $A$. thaliana seeds was significantly affected in $\triangle a b m p d$ and $\triangle a b m d h$ mutants and almost completely abolished in the $\Delta \Delta a b m p d-a b m d h$ mutants.

\section{DISCUSSION}

The main goal of this study was to specify the roles of C6-polyol mannitol in the parasitic cycle of the fungal necrotroph A. brassicicola. We showed here that fungal mannitol over-accumulated in B. oleracea leaves during the interaction with A. brassicicola, as previously reported in Arabidopsis during A. brassicicola infection (Botanga et al., 2012) or for other necrotrophic (Jobic et al., 2007; Dulermo et al., 2009) and biotrophic (Voegele et al., 2005) interactions. In the latter type of interaction, accumulated mannitol might provide a means for the fungus to store carbohydrate in a form that is not accessible to the host and maintain a gradient of metabolites in favor of the pathogen (Voegele et al., 2005). Similarly to the finding of previous studies involving fungal necrotrophs (Jobic et al., 2007; Dulermo et al., 2009), sucrose dramatically decreased during host colonization by A. brassicicola and this plant specific carbohydrate was below the detection level at $6 \mathrm{dpi}$, suggesting that mannitol biosynthesis could be a general fungal strategy to rapidly mobilize plant sugars. As proposed by Solomon et al. (2006), accumulated mannitol may then provide the necessary substrates and energy required for conidiogenesis.
Mannitol dehydrogenase (MDH), which is mainly involved in mannitol mobilization, and mannitol-1-phosphate dehydrogenase (MPD) are two key enzymes of mannitol metabolism in fungi (Krahulec et al., 2011). The corresponding A. brassicicola encoding genes ( $A b M p d$ and $A b M d h$, respectively) have been identified and their expression monitored in B. oleracea infected leaves. The AbMpd- and AbMdh-GFP fusion analysis showed that the expression pattern is closely related to the conidiation and germination processes in A. brassicicola. GFP fluorescence indicated that both proteins exhibited a relatively similar expression pattern during host infection or in vitro growth: the maximum fluorescence intensity was reached in young conidia during the conidiation process. A high fluorescence intensity was also detected in mature conidia and into the young germ tubes, whereas the signal was weaker or undetectable in mature hyphae. Our results differ from previous observations in Aspergillus niger (Aguilar-Osorio et al., 2010), reporting a spatial differentiation of the expression of these two proteins: expression of MDHA and MDH activity were detected only in spores, while expression of MPDA and MPD activity were detected only in hyphae. These conflicting results may reflect functional differences among fungal enzymes involved in mannitol metabolism.

Gradual induction of $A b M d h$ gene expression during infection was observed, as previously shown in the $U$. fabae- $V$. faba interaction (Voegele et al., 2005). The highest expression level measured was at 6 dpi, i.e., coinciding with decreased accumulation of mannitol, suggesting the mobilization of the polyol at this stage of infection may be a consequence of massive germination of newly formed conidia. It has indeed been shown in $B$. cinerea that mannitol rapidly degrades during spore germination and that such a catabolic process likely involves $\mathrm{MDH}$ activity (Dulermo et al., 2010). However, there is accumulating evidence that, contrary to what was postulated by Hult and Gatenbeck (1978), mannitol metabolism is not a cyclical process in fungi (Solomon et al., 2007; Dulermo et al., 2009). In line with this, MPD deficient A. brassisicola mutants still produced mannitol and thus AbMdh may also contribute to mannitol biosynthesis during infection. Abmpd expression was found to be induced at the earliest stages of the interaction and remained at a higher level than in control mycelia throughout the infection. According to the well-documented involvement of the MPD-dependent pathway in mannitol biosynthesis in fungi (Solomon et al., 2007), such Abmpd over-expression during tissue infection might be necessary for asexual in planta sporulation and pathogen propagation. In line with this hypothesis, $m p d$-deficient $A$. brassicicola mutants that failed to accumulate mannitol in hyphae were significantly compromised in their ability to develop conidia in planta. However, conidiation was also severely impaired in the $m d h$-deficient mutant that still accumulated high levels of intra-hyphal mannitol, thus questioning the direct relationship between mannitol content and asexual spore differentiation. Note that, as observed in B. cinerea (Dulermo et al., 2010), trehalose was detected by ${ }^{13} \mathrm{C}$ NMR in all the studied genotypes except the $m d h$-deficient mutant. This sugar might therefore also be required to promote normal conidiation in A. brassicicola, in line with its demonstrated involvement in S. nodorum sporulation (Lowe et al., 2009). 


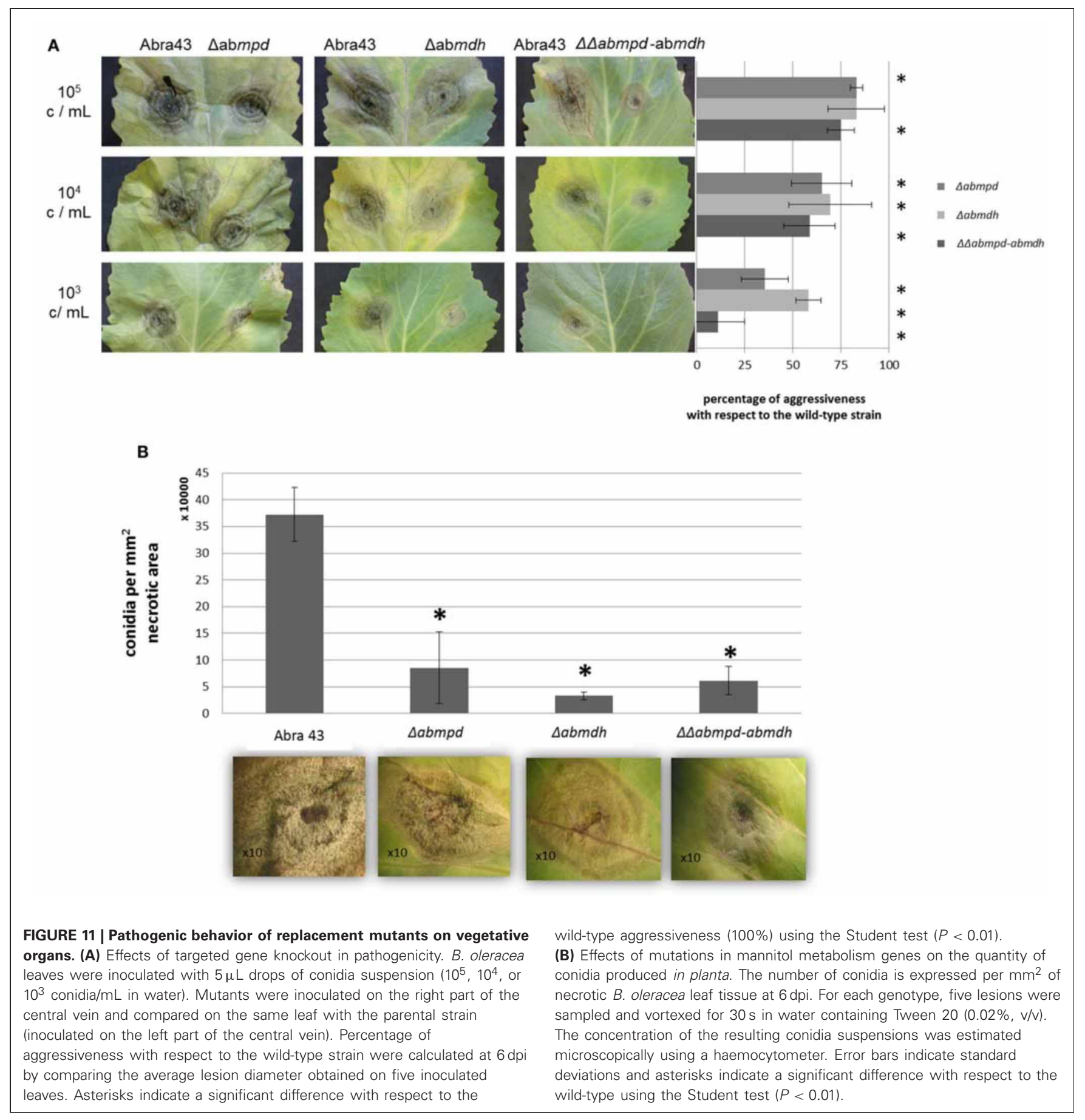

The role of stored mannitol in conidia germination also seems unclear. In conidia of all tested $A$. brassicicola mutants, a drastic decrease in mannitol to below the detectable level for the $\Delta \Delta a b m p d$-abmdh strain was observed, but normal spore germination kinetics were recorded. Similar observations were reported for A. niger and S. nodorum (Ruijter et al., 2003; Solomon et al., 2005, 2006). In contrast, the capacity to accumulate mannitol in hyphae could be correlated with the ability to differentiate penetration (i.e., appressoria-like) structures as revealed by microscopic observation of plant tissue inoculated with $m d p$ deficient mutants in which no mannitol could be detected in young hyphae. This inability to efficiently produce penetration structures by strains lacking a functional MPD-dependent pathway was not observed in A. alternata (Velez et al., 2007), but probably at least partially explained the reduced aggressiveness of A. brassicicola mpd-deficient mutants.

Besides a possible role in this pathogenesis-related developmental process, mannitol may have other functions 

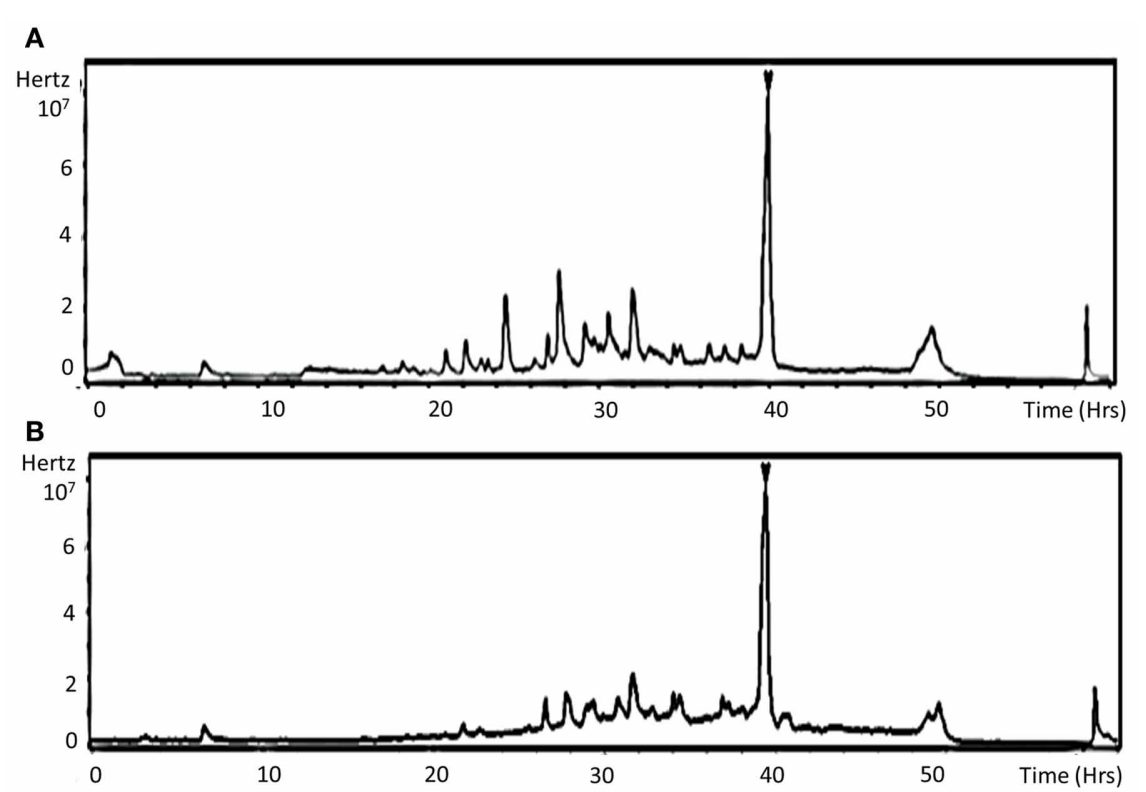

FIGURE 12 | Detection of the phytotoxin brassicicolin A in organic extracts from the culture broths of both the wild-type strain and the $\Delta \mathbf{\Delta} \mathbf{a b m p d - a b m d h}$ mutant. LC-MS total ion chromatograms
(TICs) of Abra43 (A) and $\Delta \Delta a b m p d-a b m d h$ (B) culture filtrate EtOAc extracts. Arrow indicates brassicicolin A. This experiment was done twice. during plant-fungus interactions. One earlier reported function attributed to mannitol is protection against oxidative stress generated by the host plant defense system (Jennings et al., 1998). Oxidative burst is a general plant defense mechanism that occurs at a very early stage of the interaction (Parent et al., 2008). It is characterized by rapid accumulation of hydrogen peroxide in the extracellular space of plant tissues exposed to biotic stress (Wojtaszek, 1997). This ROS, besides its potential antimicrobial activity, might regulate induced cell death at the infection site, as shown in the $A$. thaliana-A. brassicicola pathosystem (Pogany et al., 2009). In our study, A. brassicicola was found to be relatively tolerant of physiologically compatible $\mathrm{H}_{2} \mathrm{O}_{2}$ concentrations. By contrast, mannitol metabolism mutants were all characterized by increased susceptibility to this ROS and lower mannitol content in conidia. As propidium iodide staining revealed that $\mathrm{H}_{2} \mathrm{O}_{2}$ induced cell death, even in non-germinated conidia, mannitol may accumulate in this organ and have a major protective role against oxidative stress generated by $\mathrm{H}_{2} \mathrm{O}_{2}$. The decreased aggressiveness on $B$. oleracea and the lower capacity to be transmitted to Arabidopsis seeds via siliques observed for all mutant genotypes could therefore be related to their increased susceptibility to oxidative burst during the early leaf or silique infection stage. At a later infection stage, i.e., during leaf or silique tissue colonization, A. brassicicola is also exposed to glucosinolate-derived ITC that induce intracellular ROS accumulation in fungal cells (Sellam et al., 2007a). The results of the present study strongly suggested that ITC cell toxicity is mainly exerted on germ-tubes and young hyphae rather than on conidia, thus confirming previously published observations (Sellam et al., 2007b). Interestingly, $\Delta a b m p d$ and $\Delta \Delta a b m p d-a b m d h$ strains that failed to accumulate mannitol in young hyphae were found to be hyper-susceptible to allyl-, benzyl- and phenetyl-ITC and also to menadione, a reference superoxide-generating molecule. Conversely, $\Delta a b m d h$ strains that accumulated normal mannitol levels in hyphae were found to be as tolerant as the wild-type genotype. In planta assays were conducted on leaves of Brassica oleracea var. Bartolo and fruits of A. thaliana ecotype Ler that both accumulated various glucosinolates. Thus, in addition to their increased susceptibility to extracellular ROS, the low aggressiveness and seed colonization capacity of MPD-deficient mutants may also be related to their failure to overcome the intracellular oxidative stress caused by ITC during leaf or silique colonization.

Besides the capacity to colonize fruit and seed tissues, efficient seed transmission required long-term survival of the seed-borne fungus on dry seeds teguments. Ruijter et al. (2003) proposed that the alcohol functions of mannitol could enable this polyol to mimic water molecules and participate in cell tolerance to water stress. In line with this, $A$. brassicicola $\Delta \Delta a b m p d$-abmdh mutants, i.e., with no detectable hyphal and conidial mannitol, were found to be highly susceptible to matricial stress generated by PEG and showed low viability rates after 1 month of storage under on dry seeds.

The altered mannitol metabolism observed in MPD- and $\mathrm{MDH}$-deficient mutants may have other pleiotropic effects on A. brassicicola pathogenicity. During tissue invasion, necrotrophic pathogens such as A. brassicicola, synthesize phytotoxins that facilitate their spread within the infected organs (Thomma, 2003). Gloer et al. (1988) proposed that mannitol could be a precursor for the biosynthesis of brassicicolin A, which was later described as being the major host-selective phytotoxin produced by A. brassicicola (Pedras et al., 2009). Surprisingly, brassicicolin A was 


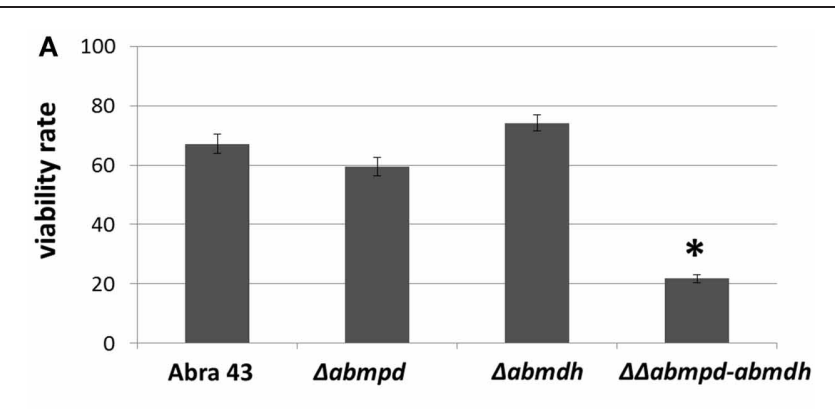

B

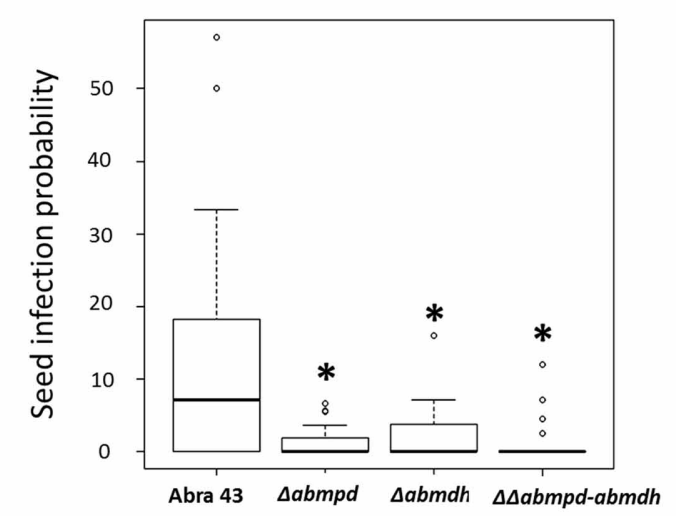

FIGURE 13 | Pathogenic behavior of replacement mutants on reproductive organs. (A) Ability of the fungus to survive after storage on dry seeds. B. oleracea seeds were artificially inoculated by incubation in a conidia suspension of wild-type or mutant strains. The fungal viability rates were estimated by calculating the ratios between the nephelometric lag times obtained from seed batches before and after 6-months of storage. Ten seeds were analyzed for each fungal genotype and the experiment was repeated twice. Error bars indicate standard deviations. Asterisks indicate a significant difference between the mutant and the parental isolate (Student test, $P<0.01$ ). (B) Influence of mannitol metabolism on the transmission capacity of $A$. brassicicola to $A$. thaliana seeds (Ler ecotype). The seed infection probability was evaluated as described by Pochon et al. (2012). The five youngest siliques of at least five plants were inoculated with each fungal genotype and the experiment was repeated twice. Contaminated siliques were harvested $10 \mathrm{dpi}$. After dissection, seeds were incubated separately on PDA medium for 2 days. A seed was considered as contaminated when incubation resulted in typical $A$. brassicicola colony development. For each inoculated fungal genotype, the seed infection probability was evaluated from at least 1000 seeds. Asterisks indicate a significant difference between the mutant and the parental isolate (Student test, $P<0.01$ ).

identified in organic extracts from the culture broths of both the wild-type strain and the $\Delta \Delta a b m p d-a b m d h$ mutant. Our HPLC

\section{REFERENCES}

Aguilar-Osorio, G., Vankuyk, P. A., Seiboth, B., Blom, D., Solomon, P. S., Vinck, A., et al. (2010). Spatial and developmental differentiation of mannitol dehydrogenase and mannitol-1-phosphate dehydrogenase in Aspergillus niger. Eukaryot. Cell 9, 1398-1402.

Botanga, C. J., Bethke, G., Chen, Z., Gallie, D. R., Fiehn, O., and

profile analysis revealed the presence of traces of mannitol in 1-week-old $\Delta \Delta a b m p d$-abmdh cultures, suggesting that mannitol was still produced in minute amounts despite complete alteration of both mannitol biosynthesis pathways. As already suggested by Dulermo et al. (2010), this latter finding challenges the existence of one additional yet undescribed pathway which could participate in mannitol metabolism and, more specifically, in A. brassicicola, in brassicicolin A synthesis. We identified several candidate enzymes to perform mannitol synthesis through potentially other metabolic routes. First, a BlastP search pointed out an A. brassicicola sequence (AB1271) sharing homology with the $\mathrm{NADP}^{+}$-dependent $\mathrm{D}$-mannitol dehydrogenase $\mathrm{TbMDH}$, described in Tuber borchii and belonging to a distinct subfamily among the polyol dehydrogenase family (Ceccaroli et al., 2007). Dulermo et al. (2010) have already suggested its involvement in B. cinerea mannitol metabolism. Secondly, we also found potential homologs of mannose-6-phosphatereductase (M6PR), a key enzyme that is involved in mannitol biosynthesis in higher plants (Everard et al., 1997).

In conclusion, these results highlight the importance of mannitol metabolism with respect to the ability of A. brassicicola to efficiently accomplish key steps of its pathogen life cycle. At the earliest stages of plant infection, the differentiation of infection structures and fungal protection against extracellular ROS generated by oxidative burst were correlated with mannitol accumulation in hyphae and conidia, respectively. During tissue colonization, although rapid conversion of plant sugars into mannitol through hyphae invasion may not be directly linked to necrotic toxin production, the polyol probably participates in fungal protection against intracellular ITC-derived oxidative stress. It may also constitute a carbohydrate store that could be remobilized during late infection stages for the in planta conidiation necessary for efficient horizontal transmission of the pathogen. Lastly, mannitol could also be involved in vertical transmission (i.e., seed transmission) of the pathogen by conferring protection against dehydration and allowing long-term survival of the fungus on stored seeds.

\section{ACKNOWLEDGMENTS}

This work was partly supported by the French Région Pays de la Loire (QUALISEM research program). We thank Stéphanie Pochon, Guillaume N'guyen, Claire Campion for help in the seed transmission assay. We are grateful to Sylvain Hanteville and Bruno Hamon, Pascale Satour, Ingrid Freuze for technical assistance. We also thank David Manley for correcting the English version of the manuscript.

utilization: a (13)C NMR study. Fungal Genet. Biol. 39, 168-175.

Ceccaroli, P., Saltarelli, R., Guescini, M., Polidori, E., Buffalini, M., Menotta, M., et al. (2007). Identification and characterization of the Tuber borchii D-mannitol dehydrogenase which defines a new subfamily within the polyol-specific medium chain dehydrogenases. Fungal Genet. Biol. 44, 965-978.
Chaturvedi, V., Flynn, T., Niehaus, W. G., and Wong, B. (1996a). Stress tolerance and pathogenic potential of a mannitol mutant of Cryptococcus neoformans. Microbiology 142(Pt 4), 937-943.

Chaturvedi, V., Wong, B., and Newman, S. L. (1996b). Oxidative killing of Cryptococcus neoformans by human neutrophils. Evidence that fungal mannitol protects 
by scavenging reactive oxygen intermediates. J. Immunol. 156, 3836-3840.

Cho, Y., Davis, J. W., Kim, K. H., Wang, J., Sun, Q. H., Cramer, R. A. Jr., et al. (2006). A high throughput targeted gene disruption method for Alternaria brassicicola functional genomics using linear minimal element (LME) constructs. Mol. Plant Microbe Interact. 19, 7-15.

Dijksterhuis, J., and de Vries, R. P. (2006). Compatible solutes and fungal development. Biochem. J. 399, e3-e5.

Dongo, A., Bataille-Simoneau, N., Campion, C., Guillemette, T., Hamon, B., Iacomi-Vasilescu, B., et al. (2009). The group III two-component histidine kinase of filamentous fungi is involved in the fungicidal activity of the bacterial polyketide ambruticin. Appl. Environ. Microbiol. 75, 127-134.

Dulermo, T., Rascle, C., Billon-Grand, G., Gout, E., Bligny, R., and Cotton, P. (2010). Novel insights into mannitol metabolism in the fungal plant pathogen Botrytis cinerea. Biochem. J. 427, 323-332.

Dulermo, T., Rascle, C., Chinnici, G., Gout, E., Bligny, R., and Cotton, P. (2009). Dynamic carbon transfer during pathogenesis of sunflower by the necrotrophic fungus Botrytis cinerea: from plant hexoses to mannitol. New Phytol. 183, 1149-1162.

Ehrenshaft, M., and Upchurch, R. (1993). Host protein(s) induces accumulation of toxin cercosporin and mRNA in phytopathogenic strain of Cercospora kikuchkii. Physiol. Mol. Plant Pathol. 43, 95-101.

Everard, J. D., Cantini, C., Grumet, R., Plummer, J., and Loescher, W. H. (1997). Molecular cloning of mannose-6-phosphate reductase and its developmental expression in celery. Plant Physiol. 113, 1427-1435.

Gloer, J. B., Poch, G. K., Short, D. M., and McCloskey, D. V. (1988). Structure of brassicicolin A: a isocyanide antibiotic from the phylloplane fungus Alternaria brassicicola. J. Organ. Chem. 53, 3758-3761.

Hoch, H. C., Galvani, C. D., Szarowski, D. H., and Turner, J. N. (2005). Two new fluorescent dyes applicable for visualization of fungal cell walls. Mycologia 97, 580-588.

Horer, S., Stoop, J., Mooibroek, H., Baumann, U., and Sassoon, J. (2001). The crystallographic structure of the mannitol 2dehydrogenase NADP+ binary complex from Agaricus bisporus. J. Biol. Chem. 276, 27555-27561.
Hult, K., and Gatenbeck, S. (1978). Production of NADPH in the mannitol cycle and its relation to polyketide formation in Alternaria alternata. Eur. J. Biochem. 88, 607-612.

Iacomi-Vasilescu, B., BatailléSimoneau, N., Campion, C., Dongo, A., Laurent, E., Serandat, I., et al. (2008). Effect of null mutations in the AbNIK1 gene on saprophytic and parasitic fitness of Alternaria brassicicola isolates highly resistant to dicarboximides fungicides. Plant Pathol. 57, 937-947.

Jennings, D. B., Daub, M. E., Pharr, D. M., and Williamson, J. D. (2002). Constitutive expression of a celery mannitol dehydrogenase in tobacco enhances resistance to the mannitol-secreting fungal pathogen Alternaria alternata. Plant J. 32, 41-49.

Jennings, D. B., Ehrenshaft, M., Pharr, D. M., and Williamson, J. D. (1998) Roles for mannitol and mannitol dehydrogenase in active oxygenmediated plant defense. Proc. Natl. Acad. Sci. U.S.A. 95, 15129-15133.

Jobic, C., Boisson, A. M., Gout, E., Rascle, C., Fevre, M., Cotton, P., et al. (2007). Metabolic processes and carbon nutrient exchanges between host and pathogen sustain the disease development during sunflower infection by Sclerotinia sclerotiorum. Planta 226, 251-265.

Jornvall, H., Persson, B., Krook, M., Atrian, S., Gonzalez-Duarte, R., Jeffery, J., et al. (1995). Short-chain dehydrogenases/reductases (SDR). Biochemistry 34, 6003-6013.

Joubert, A., Bataille-Simoneau, N., Campion, C., Guillemette, T. Hudhomme, P., Iacomi-Vasilescu, B., et al. (2011a). Cell wall integrity and high osmolarity glycerol pathways are required for adaptation of Alternaria brassicicola to cell wall stress caused by brassicaceous indolic phytoalexins. Cell. Microbiol. 13, 62-80.

Joubert, A., Simoneau, P., Campion, C., Bataille-Simoneau, N., IacomiVasilescu, B., Poupard, P., et al. (2011b). Impact of the unfolded protein response on the pathogenicity of the necrotrophic fungus Alternaria brassicicola. Mol. Microbiol. 79, 1305-1324.

Joubert, A., Calmes, B., Berruyer, R., Pihet, M., Bouchara, J. P., Simoneau, P., et al. (2010). Laser nephelometry applied in an automated microplate system to study filamentous fungus growth. Biotechniques 48, 399-404.

Juchaux-Cachau, M., LandouarArsivaud, L., Pichaut, J. P., Campion, C., Porcheron,
B., Jeauffre, J., et al. (2007). Characterization of AgMaT2, a plasma membrane mannitol transporter from celery, expressed in phloem cells, including phloem parenchyma cells. Plant Physiol. 145, 62-74.

Krahulec, S., Armao, G. C., Klimacek, M., and Nidetzky, B. (2011). Enzymes of mannitol metabolism in the human pathogenic fungus Aspergillus fumigatus-kinetic properties of mannitol-1-phosphate 5-dehydrogenase and mannitol 2-dehydrogenase, and their physiological implications. FEBS J. 278, 1264-1276.

Lewis, D., and Smith, D. (1967). Sugar alcohols in fungi and green plants. New Phytol. 66, 143-184.

Lorang, J. M., Tuori, R. P., Martinez, J. P., Sawyer, T. L., Redman, R. S., Rollins, J. A., et al. (2001). Green fluorescent protein is lighting up fungal biology. Appl. Environ. Microbiol. 67, 1987-1994.

Lowe, R. G., Lord, M., Rybak, K., Trengove, R. D., Oliver, R. P., and Solomon, P. S. (2009). Trehalose biosynthesis is involved in sporulation of Stagonospora nodorum. Fungal Genet. Biol. 46, 381-389.

Malonek, S., Rojas, M. C., Hedden, P., Gaskin, P., Hopkins, P., and Tudzynski, B. (2004). The NADPHcytochrome $\mathrm{P} 450$ reductase gene from Gibberella fujikuroi is essential for gibberellin biosynthesis. J. Biol. Chem. 279, 25075-25084.

Parent, C., Capelli, N., and Dat, J. (2008). Reactive oxygen species, stress and cell death in plants. C. $R$. Biol. 331, 255-261.

Pedras, M. S. C., Chumala, P. B., Jin, W., Islam, M. S., and Hauck, D. W. (2009). The phytopathogenic fungus Alternaria brassicicola: phytotoxin production and phytoalexin elicitation. Phytochemistry 70, 394-402.

Pedras, M. S. C., Khan, A. Q., Smith, K. C., and Stettner, S. L. (1997). Preparation, biotransformation, and antifungal activity of methyl benzyldithiocarbamates. Can. J. Chem. 75, 825-828.

Pochon, S., Terrasson, E., Guillemette, T., Iacomi-Vasilescu, B., Georgeault, S., Juchaux, M., et al. (2012). The Arabidopsis thaliana - Alternaria brassicicola pathosystem: a model interaction for investigating seed transmission of necrotrophic fungi. Plant Methods 8:16. doi: 10.1186/1746-4811-8-16

Pogany, M., Von Rad, U., Grun, S., Dongo, A., Pintye, A., Simoneau, P., et al. (2009). Dual roles of reactive oxygen species and $\mathrm{NADPH}$ oxidase RBOHD in an ArabidopsisAlternaria pathosystem. Plant physiol. 151, 1459-1475.

Rosnoblet, C., Aubry, C., Leprince, O., Vu, B. L., Rogniaux, H., and Buitink, J. (2007). The regulatory gamma subunit SNF4b of the sucrose non-fermenting-related kinase complex is involved in longevity and stachyose accumulation during maturation of Medicago truncatula seeds. Plant J. 51, 47-59.

Ruijter, G. J., Bax, M., Patel, H., Flitter, S. J., van de Vondervoort, P. J., de Vries, R. P., et al. (2003). Mannitol is required for stress tolerance in Aspergillus niger conidiospores. Eukaryot. Cell 2, 690-698.

Sellam, A., Dongo, A., Guillemette, T., Hudhomme, P., and Simoneau, P. (2007a). Transcriptional responses to exposure to the brassicaceous defence metabolites camalexin and allyl-isothiocyanate in the necrotrophic fungus Alternaria brassicicola. Mol. Plant Pathol. 8, 195-208.

Sellam, A., Iacomi-Vasilescu, B. Hudhomme, P., and Simoneau, P. (2007b). In vitro antifungal activity of brassinin, camalexin and two isothiocyanates against the crucifer pathogens Alternaria brassicicola and Alternaria brassicae. Plant Pathol. 56, 296-301.

Shen, B., Jensen, R. G., and Bohnert, H. J. (1997a). Increased resistance to oxidative stress in transgenic plants by targeting mannitol biosynthesis to chloroplasts. Plant Physiol. 113, 1177-1183.

Shen, B., Jensen, R. G., and Bohnert, H. J. (1997b). Mannitol protects against oxidation by hydroxyl radicals. Plant Physiol.115, 527-532.

Smirnoff, N., and Cumbes, Q. J. (1989). Hydroxyl radical scavenging activity of compatible solutes. Phytochemistry 28, 1057-1060.

Solomon, P. S., Tan, K. C., and Oliver, R. P. (2005). Mannitol 1phosphate metabolism is required for sporulation in planta of the wheat pathogen Stagonospora nodorum. Mol. Plant Microbe Interact.18, 110-115.

Solomon, P. S., Waters, O. D., Jorgens, C. I., Lowe, R. G., Rechberger, J., Trengove, R. D., et al. (2006). Mannitol is required for asexual sporulation in the wheat pathogen Stagonospora nodorum (glume blotch). Biochem. J. 399, 231-239.

Solomon, P. S., Waters, O. D., and Oliver, R. P. (2007). Decoding the mannitol enigma in filamentous fungi. Trends Microbiol. 15, 257-262. 
Stoop, J., and Pharr, D. M. (1993). Effect of different carbon sources on relative growth rate, internal carbohydrates, and mannitol 1oxidoreductase activity in celery suspension cultures. Plant Physiol. 103, 1001-1008.

Sweigard, J. A., Carroll, A. M., Kang, S., Farrall, L., Chumley, F. G., and Valent, B. (1995). Identification, cloning, and characterization of PWL2, a gene for host species specificity in the rice blast fungus. Plant Cell 7, 1221-1233.

Thomma, B. P. (2003). Alternaria spp.: from general saprophyte to specific parasite. Mol. Plant Pathol. 4, 225-236.

Velez, H., Glassbrook, N. J., and Daub, M. E. (2007). Mannitol metabolism in the phytopathogenic fungus Alternaria alternata. Fungal Genet. Biol. 44, 258-268.
Velez, H., Glassbrook, N. J., and Daub, M. E. (2008). Mannitol biosynthesis is required for plant pathogenicity by Alternaria alternata. FEMS Microbiol. Lett. 285, 122-129.

Verslues, P. E., Agarwal, M., KatiyarAgarwal, S., Zhu, J., and Zhu, J. K. (2006). Methods and concepts in quantifying resistance to drought, salt and freezing, abiotic stresses that affect plant water status. Plant J. 45, 523-539.

Voegele, R. T., Hahn, M., Lohaus, G. Link, T., Heiser, I., and Mendgen, K. (2005). Possible roles for mannitol and mannitol dehydrogenase in the biotrophic plant pathogen Uromyces fabae. Plant Physiol. 137, 190-198.

Vogel, H. J. (1956). A convenient growth medium for Neurospora crassa. Microbial Genet. Bull. 13, 42-43.
Wojtaszek, P. (1997). Oxidative burst: an early plant response to pathogen infection. Biochem. J. 322( $\mathrm{Pt} \mathrm{3}$, 681-692.

Yu, J. H., Hamari, Z., Han, K. H., Seo, J. A., Reyes-Dominguez, Y., and Scazzocchio, C. (2004). Doublejoint PCR: a PCR-based molecular tool for gene manipulations in filamentous fungi. Fungal Genet. Biol. 41, 973-981.

Conflict of Interest Statement: The authors declare that the research was conducted in the absence of any commercial or financial relationships that could be construed as a potential conflict of interest.

Received: 28 January 2013; accepted: 20 April 2013; published online: 13 May 2013.
Citation: Calmes B, Guillemette T, Teyssier L, Siegler B, Pigné S, Landreau $A$, Iacomi B, Lemoine R, Richomme $P$ and Simoneau $P$ (2013) Role of mannitol metabolism in the pathogenicity of the necrotrophic fungus Alternaria brassicicola. Front. Plant Sci. 4:131. doi: 10.3389/fpls.2013.00131

This article was submitted to Frontiers in Plant-Microbe Interaction, a specialty of Frontiers in Plant Science.

Copyright (c) 2013 Calmes, Guillemette, Teyssier, Siegler, Pigné, Landreau, Iacomi, Lemoine, Richomme and Simoneau. This is an open-access article distributed under the terms of the Creative Commons Attribution License which permits use, distribution and reproduction in other forums, provided the original authors and source are credited and subject to any copyright notices concerning any third-party graphics etc. 